Sādhanā Vol. 39, Part 6, December 2014, pp. 1471-1495. (C) Indian Academy of Sciences

\title{
Rheological behaviour of self-compacting micro-concrete
}

\section{BURAK FELEKOĞLU}

Dokuz Eylul University, Faculty of Engineering, Civil Engineering Department, Tinaztepe Campus, 35397 Buca, İzmir, Turkey

e-mail: burak.felekoglu@deu.edu.tr

MS received 12 January 2014; revised 28 April 2014; accepted 11 June 2014

\begin{abstract}
The rheological behaviour of Self-Compacting Micro-Concrete (SCMC) mixtures has been investigated within the scope of this paper. Rheological measurements have been performed using a novel rheometer equipped with a ball measuring system. Three SCMC mixtures with the same water/cement (W/C) ratios $(0.44$ by weight) and cement/limestone powder (1/1 by weight) with different High Range Water Reducing Admixtures (HRWRA) contents (1, 1.2 and $1.4 \%$ by weight of cement) have been tested. For comparison purpose, three conventional cement paste mixtures with varying $\mathrm{W} / \mathrm{C}$ ratios $(0.30,0.325$ and 0.35$)$ were also prepared. Flow parameters such as yield value and plastic viscosity have been measured along with shear thickening or thinning behaviour. Furthermore, thixotropic behaviour of the various mixtures have been measured using hysteresis areas. Finally, advanced rheological properties of micro-concretes and cement pastes have been compared and discussed. While cement pastes investigated in this study possessed high yield values (34-217 Pa) and very low viscosities (5-19 Pa.s), comparatively high viscosities (1745 Pa.s) and low yield values (0-47 Pa) have been obtained from SCMCs. The high viscosity of micro-concretes improves the solid holding capacity of this composite. According to flow curve analysis, cement paste mixtures and SCMCs exhibited shear thinning and shear thickening characteristics, respectively.
\end{abstract}

Keywords. Workability; viscosity; cement paste; high range water reducing admixture.

\section{Introduction}

There is an increasing tendency of using smaller maximum aggregate sizes in the design of micro-concrete mixtures, such as engineered cementitious composites (ECC) and other high performance fibre reinforced cement-based composites (HPFRCC). Self-compacting microconcrete (SCMC) can be defined as a mixture of binders and inert materials with a maximum aggregate size smaller than $100 \mu \mathrm{m}$ that has a flowable and non-segregate cohesive consistency (Felekoglu 2009). The rheology of SCMC can be tailored by balancing the high range water reducing admixture (HRWRA) dosage and water content. While SCMC can be applied as grout material for injection purposes for its high filling capacity, its application without fibre reinforcement is limited for other areas due to its brittle structure. Li (2002) and Naaman \& 
Reinhardt (2006) reported that incorporation of polymeric or specially designed metallic fibres at varying dosages may considerably improve their ductility by exhibiting controlled multiple micro-cracks. According to Li et al (2002) these composites display extraordinary high tensile strain capacities. For example, ECC mixture incorporating approximately 1.5-2\% PVA (Polyvinyl alcohol) fibres by volume exhibited a tensile strain capacity of $2.5-4 \%$. In addition to fibre incorporation, many of the recently developed high performance cementitious composite applications deal with two main matrix phase modifications: the first one is the increase of the amount of paste phase (and simultaneously reduction of maximum aggregate size); the second one is the increase of the workability by the incorporation of polycarboxylate based HRWRA as reported by Li et al (1998). According to Sahmaran et al (2009), self-compacting cementitious mixture with smaller maximum aggregate sizes have been gaining popularity and usually chosen to ease and fasten the casting of structures. From the scientific point of view, SCMC can also be considered as the paste phases of self-compacting concrete (SCC) and high performance concrete (HPC) applications (Kong et al 2003). However, recent studies by Barrak et al (2009) showed that rheological behaviour of SCMC is significantly different from ordinary cement paste. The addition of specific ingredients to SCMC strongly alters its rheological properties (Yang et al 2009). Mazanec et al (2010) experimentally showed the significance of matrix paste phase composition to link fresh concrete workability and mixing intensity.

In this paper, rheological measurements have been performed using a novel rheometer equipped with a ball measuring system. SCMC mixtures with various HRWRA contents and conventional cement paste mixtures with varying water/cement ratios were prepared. Flow parameters such as yield value and plastic viscosity have been measured along with the shear thickening or thinning behaviour. Furthermore, thixotropic behaviour of the various mixtures have been measured using hysteresis areas between ascending and descending flow curves. The advanced rheological properties of micro-concretes and cement pastes have been compared and discussed in detail.

\section{Experimental study}

\subsection{Materials}

Ordinary Portland cement (CEM I 42.5R) have been procured from Çimentaş Cement Plant. The Bogue composition of cement determined from X-ray fluorescence analysis (weight \%), was: $\mathrm{C}_{3} \mathrm{~S}(66.99 \%), \mathrm{C}_{2} \mathrm{~S}(2.96 \%), \mathrm{C}_{3} \mathrm{~A}(8.01 \%), \mathrm{C}_{4} \mathrm{AF}(10.38 \%)$. The Blaine surface area and specific gravity of cement were $370 \mathrm{~m}^{2} / \mathrm{kg}$ and 3.12 , respectively. Limestone powder which was collected from the filtration system of a quarry crusher has been used as micro-aggregate. According to chemical analysis, the $95.4 \%$ of this powder was $\mathrm{CaCO}_{3}$ with impurities including: $\mathrm{Al}_{2} \mathrm{O}_{3}$ less than $0.1 \% ; \mathrm{MgCO}_{3}, 0.5 \% ; \mathrm{Fe}_{2} \mathrm{O}_{3}, 0.3 \%$ and $\mathrm{SiO}_{2}, 0.8 \%$. The Blaine specific surface area value and specific gravity of this powder were $443 \mathrm{~m}^{2} / \mathrm{kg}$ and 2.58, respectively (Felekoglu 2007).

In order to improve the flowability of micro-concretes, a polycarboxylate based HRWRA with an acrylic copolymer structure (acrylic backbone grafted by polyoxyethylene side-chains) have been used. The side-chains of this specially designed admixture were bonded to the main chain by ester bond. A detailed characterization of this admixture can be found in Felekoglu \& Sarıkahya (2008). 


\subsection{Mixture proportions and preparation of samples}

Three micro-concrete (MC) mixtures with the same water/cement ratios ( 0.44 by weight) and cement/limestone powder (1/1 by weight) with different HRWRA contents $(1,1.2$ and $1.4 \%$ by weight of cement) have been prepared.These mixture proportions were selected after performing a set of pre-trial tests and details are reported in Felekoglu (2009). The cement, limestone powder and water contents of 1 cubic meter of mixture are $872 \mathrm{~kg}, 872 \mathrm{~kg}$ and $384 \mathrm{~kg}$, respectively. Three conventional cement paste mixtures with varying W/C ratios $(0.30,0.325$ and 0.35$)$ were also tested for comparison purpose.

Fresh state conditions of mixtures have been illustrated in figure 1. Spread flow tests were performed with a mini-slump cone mold conforming ASTM C230 standard. Fresh mixture is filled to the cone without any compaction and then the cone was lifted up and final spread diameters are photographed. These images can give an idea about the consistency of the mixtures. As expected, the workability of cement pastes improved by increasing the W/C ratios. On the other side, MC mixture can be compared qualitatively as follows: while MC-1.0 mixture was cohesive and slow flowing, MC-1.4 mixture had a quick spreading self-flowable structure. When compared to MC-1.4 mixture, MC-1.2 was more stable in terms of consistency. The initial spread flow values of MC-1.0, MC-1.2 and MC-1.4 mixture were 210, 310 and $350 \mathrm{~mm}$, respectively. These flow values were determined by using a spread cone $(50 \mathrm{~mm}$ height, 70 and $100 \mathrm{~mm}$ of upper and lower cone diameter conforming ASTM C230) without any external vibration. The HRWRA dosages were between the maximum (1.4\%) and minimum limits (1.0\%) in terms of self-flowability and stability (segregation and bleeding resistance ability).

Mixture was prepared by using a Hobart mixer at controlled laboratory conditions. Different mixing procedures were applied for cement paste and micro-concrete mixtures. Cement paste mixture has been prepared in 3 minutes. Cement and water was mixed for 1 minute at $62 \mathrm{rpm}$ and 2 minutes at $125 \mathrm{rpm}$. On the other hand, preparation time of micro-concrete mixture was 3.5 minutes. Cement and limestone powder dry was mixed for 30 seconds at $62 \mathrm{rpm}$. Gradually water was added and mixed for 1 minute at the same speed. Finally, HRWRA introduced to

\section{Cement paste series}

$\mathrm{CP}-\mathbf{0 . 3 0} 0^{\wedge}$

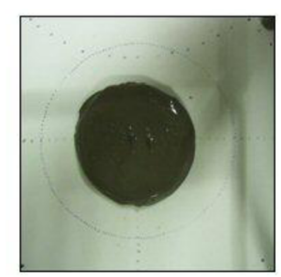

CP- $0.325^{*}$

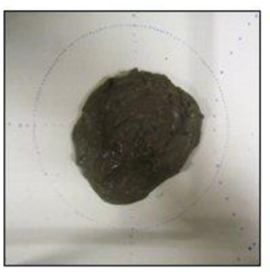

CP-0.35*

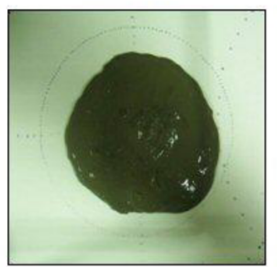

Micro concrete series

MC-1.0" (cohesive)

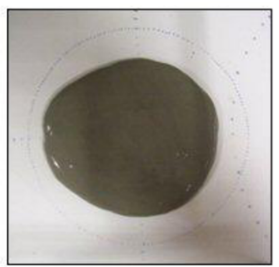

MC-1.2** (stable)

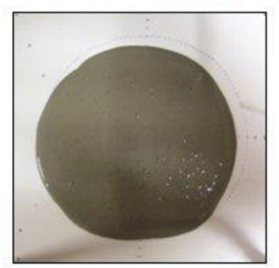

MC $-1.4^{* *}$ (highly flowable)

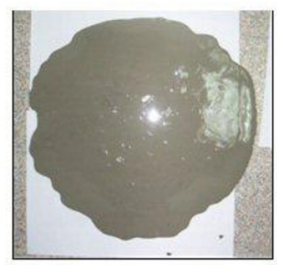

Figure 1. Fresh state images of mixture after rheological testing (frame size of each photograph: $400 \times$ $400 \mathrm{~mm}) . * \mathrm{~W} / \mathrm{C}$ ratios, **Superplasticizer dosages (\% by weight of cement weight). 
the wet mixture and mixing was performed at high speed $(125 \mathrm{rpm})$ for 2 minutes. The aim of applying the high speed mixing was to improve the effectiveness of polycarboxylate-based HRWRAs long chain molecules by orienting them parallel to mixture flow stream. At the end of this mixing procedure, mixture with an approximate effective volume of $400 \mathrm{~cm}^{3}$ were poured into the container of the rheometer. While the flowable mixtures (SCMCs) with self-leveling property just poured into container, compaction was needed for less workable cement pastes and they were tilted.

\subsection{Experimental set-up and measuring procedures}

Rheological measurements have been performed by using a Physica (Anton Paar) MCR 51 rheometer equipped with a ball measuring system (BMS). Maximum allowable shear rate was $35 \mathrm{~s}^{-1}$ (60 rpm for $8 \mathrm{~mm} \mathrm{BMS}$ ) and maximum torque capacity of the rheometer was $2850 \mathrm{~Pa}$. BMS was specially developed for cement pastes and mortars by Schatzmann et al (2004) and Kaufmann et al (2006). Drag resistance of the ball buried in the sample have been determined as a function of rotational speed. These data then converted to shear stress versus shear rate by using Schatzmann's (2005) empirical conversion constants. Detailed theoretical background for this measuring system can be found in Schatzmann et al (2003). The diameter of the ball used in this application was $8 \mathrm{~mm}$ and measuring system can be seen in figure 2. Measurements have been conducted at constant temperature $\left(20 \pm 1^{\circ} \mathrm{C}\right)$ with the help of a water bath assembled to the rheometer container (Anton Paar Viscotemp 2 VT-2). Rheoplus software has been used for data processing and modelling applications. It is possible to program the rheometer by using macros for the application of any shear rate protocol. Shear rate protocol used in this study have been presented in figure 3. A comprehensive shear rate protocol was used in order to investigate the role of shear rate change at different time intervals (the speed of shear rate change). Another aim of using such a shear rate protocol was to investigate the accuracy of test results at different shear rate accelerations. The shear rate protocol has been divided into 18 sections. Sections $1-2,7-8$ and 13-14 have been prepared to erase the shear history of mixture and no data are collected through these stages. Other sections consist of increasing and decreasing shear rate ramps. Three different time intervals have been selected for each group of ramps in order to compare the

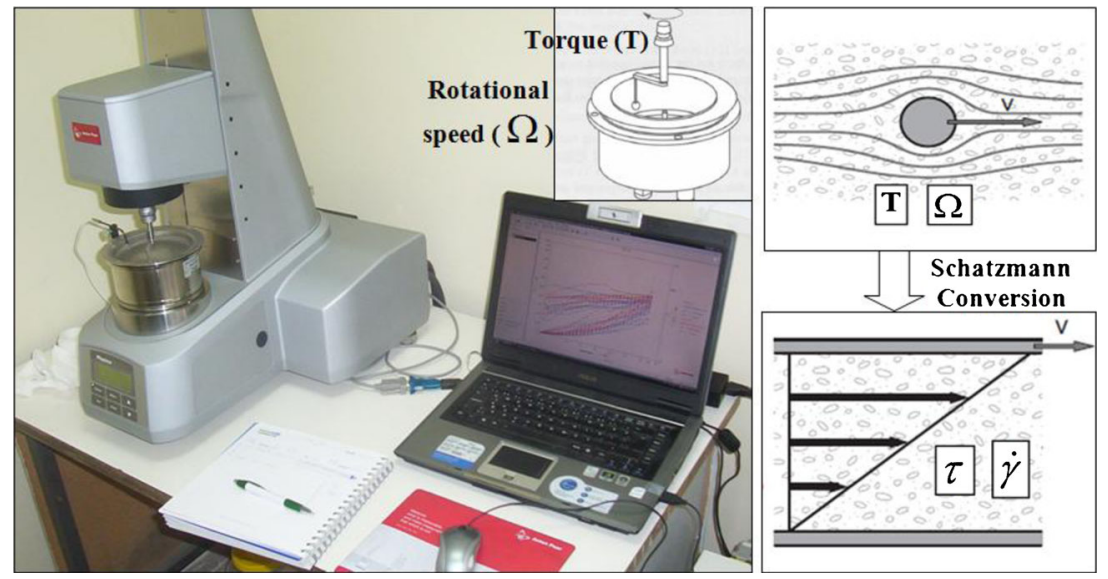

Figure 2. Physica (Anton Paar) MCR 51 rheometer equipped with a ball measuring system (BMS) and schematic representation of Schatzmann conversion (Schatzmann 2005). 


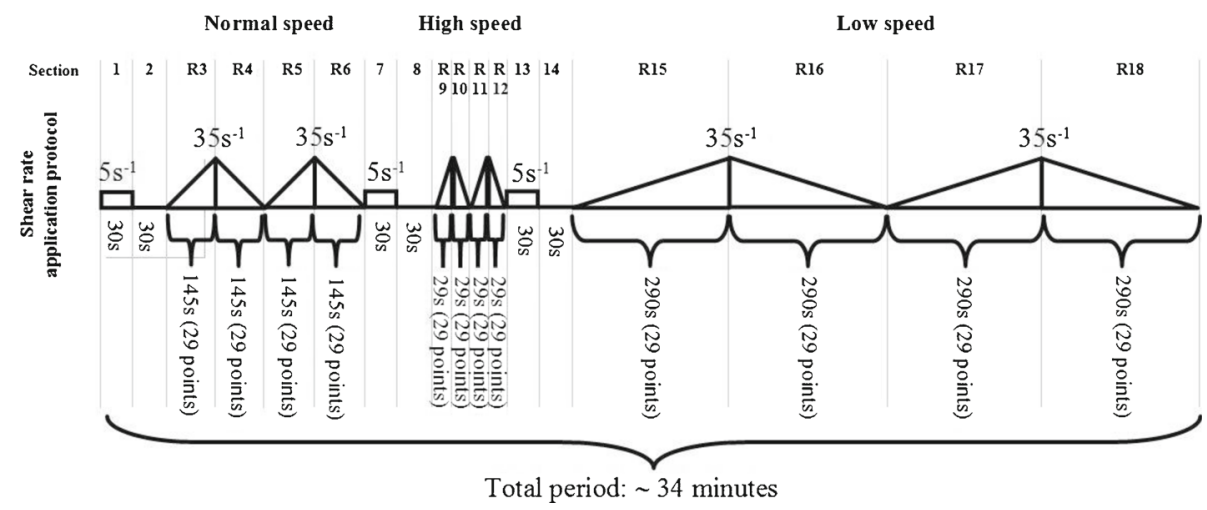

Figure 3. Shear rate application protocol used in this study. (normal speed: time interval between minimum and maximum shear rate is $145 \mathrm{~s}$., high speed: time interval between minimum and maximum shear rate is 29 s., low speed: time interval between minimum and maximum shear rate is 290s.)

behaviour of $\mathrm{CP}$ and MC mixtures subjected to types of applications (including initial and final moments of spraying, pumping and pouring, etc.). The period of increasing shear rate from 1 to $35 \mathrm{~s}^{-1}$ were 145, 29 and 290 seconds for normal, high and low speed of shear rate change. Total period of shear rate protocol was approximately 34 minutes and 348 flow curve points have been
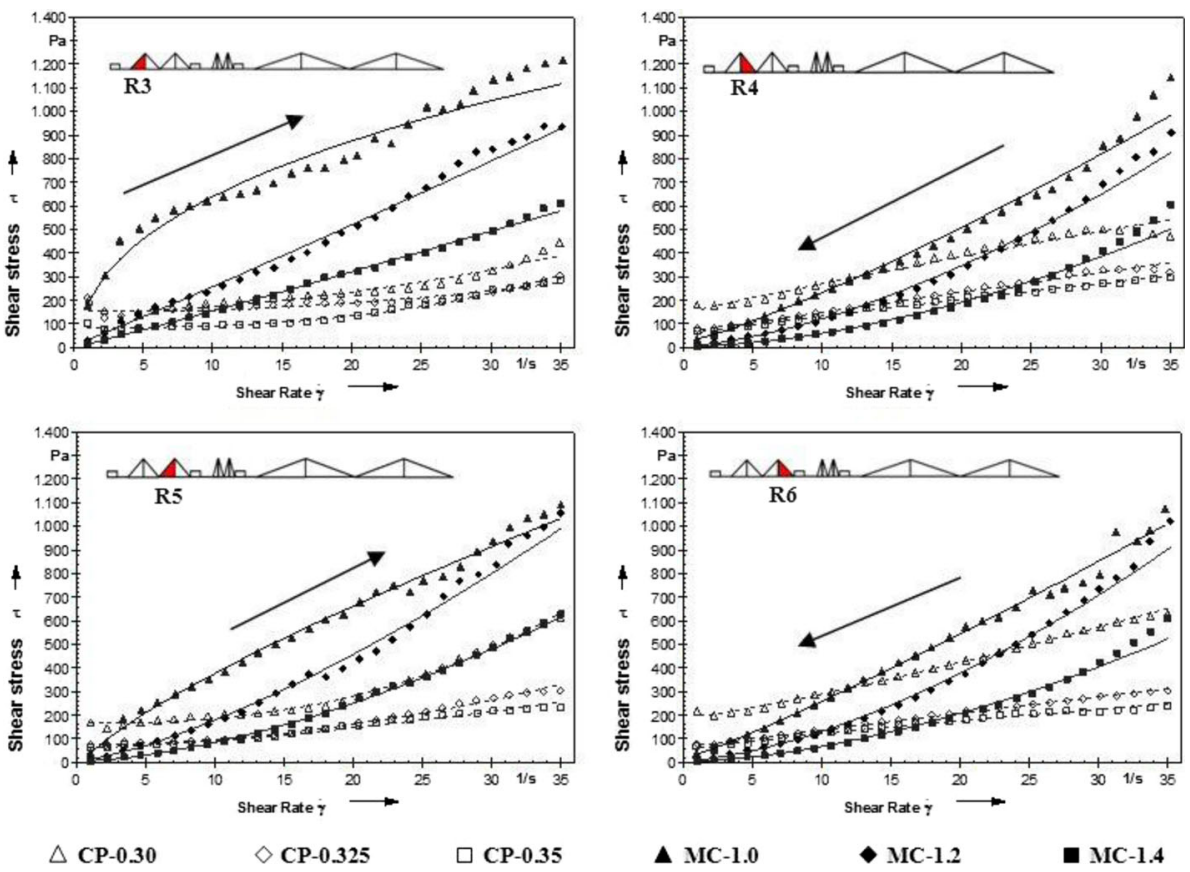

$\Delta \mathrm{MC}-1.0$

$\bullet \mathrm{MC}-1.2$

MC-1.4

Figure 4. Flow curves of each mixture at the normal speed ramps group (R3-R4-R5-R6). (CP: cement paste, 0.30-0.325-0.35 refer to water/cement ratio, MC: micro-concrete, 1.0-1.2-1.4 refer to superplasticizer dosage by weight of cement.) 
collected for each mixture. The flow curves were then measured and rheological parameters have been computed for all ascending and descending intervals of the protocol.

BMS is a comparatively new and different rheometer system. The comparison of this rheometer with conventional rheometers has been studied by Schatzmann (2005). Additionally, readers interested in the comparison of different rheometers can refer to the study of Feys et al (2009). In that study, the rheological properties of traditional cement pastes and paste phases of different SCC's had been investigated by using a conventional rheometer with a plate-plate geometry.

\subsection{Rheological modelling of flow curves and determination of rheological parameters}

Flow curves of mixtures at normal, high and low speed of shear rate application ramps have been presented in figures 4-6, respectively. Graphics have been grouped according to the speed of shear rate change. In these figures, the raw data (shear stress $(\mathrm{Pa})$ versus shear rate $\left(\mathrm{s}^{-1}\right)$ ) obtained from rheometer have been presented by using different symbols (solid triangle: MC1.0, solid diamond: MC-1.2, solid square: MC-1.4, hollow triangle: CP-0.30, hollow diamond: CP-0.325, hollow square: CP-0.35). Flow curves have been plotted by using Herschel Bulkley model as similar to the reported in Mouret \& Cyr (2003). Before selecting this model, different rheological models which can be found in Papo \& Piani's work (2004), have been studied (Bingham, Herschel Bulkley, Casson, Quemada, Papo \& Piani models, etc.). HerschelBulkley model was found as the most suitable model to characterize the shear rate dependent
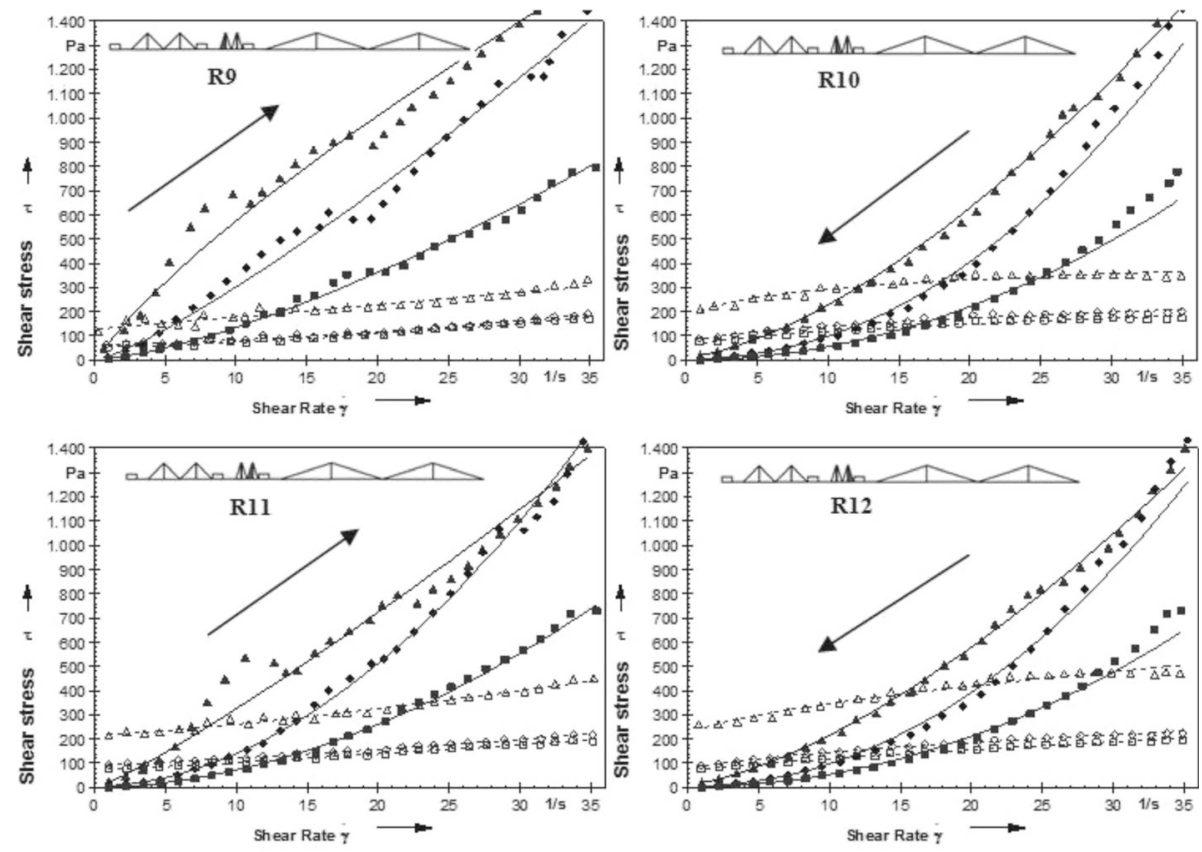

$\triangle \mathrm{CP}-0.30$

$\diamond \mathrm{CP}-0.325$

$\square$ CP- 0.35

A MC-1.0

- MC-1.2

- MC-1.4

Figure 5. Flow curves of each mixture at the high speed ramps group (R9-R10-R11-R12). (CP: cement paste, 0.30-0.325-0.35 refer to water/cement ratio, MC: micro-concrete, 1.0-1.2-1.4 refer to superplasticizer dosage by weight of cement). 

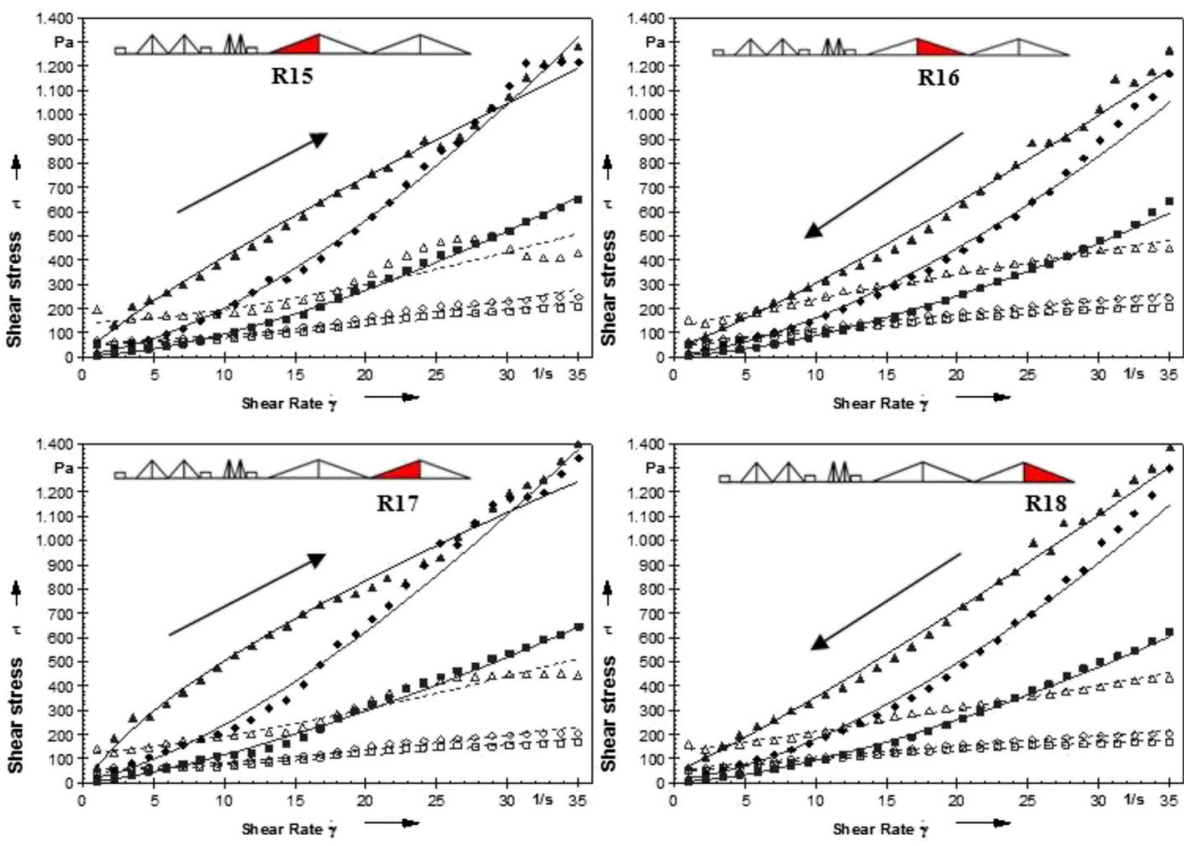

$\triangle \mathrm{CP}-0.30$

$\diamond \mathrm{CP}-0.325$

$\square$ CP-0.35

$\triangle \mathrm{MC}-1.0$

- MC-1.2

MC-1.4

Figure 6. Flow curves of each mixture at the low speed ramps group (R15-R16-R17-R18). (CP: cement paste, 0.30-0.325-0.35 refer to water/cement ratio, MC: micro-concrete, 1.0-1.2-1.4 refer to superplasticizer dosage by weight of cement).

viscosity variation of self-flowing cementitious mixtures. This result was in accordance with previous investigations conducted by Ferraris (1999). The Herschel-Bulkley model describes the flow curve of a material with a yield stress and shear thinning or shear thickening behaviour at stresses above the yield stress. Model formulae can be given as follows

$$
\tau=\tau_{0}+b \dot{\gamma}^{p},
$$

where $\tau$ : shear stress $(\mathrm{Pa})$ at any shear rate (y-axis variable), $\tau_{o}$ : yield value $(\mathrm{Pa}), \dot{\gamma}$ : Shear rate (1/s) (x-axis variable), $\mathrm{b}$ and $\mathrm{p}$ are model coefficients. The yield values have been calculated as yield stress values at zero shear rate and have been presented in table 1 . Power coefficient $\mathrm{p}$, is an indicator of the viscosity change as a function of shear rate. If $\mathrm{p}>1$ shear thickening behaviour is dominant. On the contrary, if $\mathrm{p}<1$ shear thinning behaviour is dominant. Finally, viscosity is independent from shear rate if $p=1$. Banfill \& Frias (2007) and Azeredo et al (2008), preferred Bingham Model to simulate the rheology of cement paste. Since the Bingham model is a special case of Herschel-Bulkley Model (p $=1$ ), both cement pastes and micro-concretes can be modelled by Herschel-Bulkley formulae. In case of Binghamian materials, viscosity is constant. This constant viscosity value derived from Bingham model is called 'plastic viscosity $(\mu)$ ' as suggested by Koehler \& Fowler (2004). On the other hand, according to Herschel-Bulkley Model there is no constant viscosity value. The shear rate dependent viscosity values of most Herschel-Bulkley 
Table 1. Yield values $\left(\tau_{o}\right)$ of mixtures determined by Herschel-Bulkley analyses (Pa).

\begin{tabular}{|c|c|c|c|c|c|c|c|c|c|c|c|c|}
\hline \multirow{3}{*}{$\begin{array}{l}\text { Section no. } \\
\text { Mixture code }\end{array}$} & 3 & 4 & 5 & 6 & 9 & 10 & 11 & 12 & 15 & 16 & 17 & 18 \\
\hline & \multicolumn{4}{|c|}{$\begin{array}{c}\text { Shear rate acceleration: } \\
\text { normal }\end{array}$} & \multicolumn{4}{|c|}{$\begin{array}{c}\text { Shear rate acceleration: } \\
\text { high }\end{array}$} & \multicolumn{4}{|c|}{$\begin{array}{c}\text { Shear rate acceleration: } \\
\text { low }\end{array}$} \\
\hline & \multicolumn{4}{|c|}{$(\mathrm{Pa})$} & \multicolumn{4}{|c|}{$(\mathrm{Pa})$} & \multicolumn{4}{|c|}{$(\mathrm{Pa})$} \\
\hline CP-0.30 & 152 & 141 & 166 & 190 & 129 & 67 & 217 & 215 & 142 & 114 & 123 & 131 \\
\hline CР-0.325 & 151 & 66 & 75 & 64 & 67 & 61 & 94 & 72 & 60 & 48 & 55 & 47 \\
\hline CP-0.35 & 85 & 55 & 62 & 49 & 56 & 49 & 71 & 65 & 49 & 37 & 45 & 34 \\
\hline MC-1.0 & -* & 25 & $-*$ & 17 & -* & 16 & -* & 15 & 3 & 40 & -* & 47 \\
\hline MC- 1.2 & 11 & 8 & 7 & 8 & $-*$ & 7 & 5 & 7 & 12 & 12 & 14 & 15 \\
\hline MC-1.4 & 2 & 5 & 5 & 4 & 2 & 2 & 2 & 4 & 4 & 5 & 4 & 4 \\
\hline
\end{tabular}

*Mixtures presenting negative yield values (physically meaningless) according to Herschel-Bulkley model analysis.

mixtures have become stationary at very high shear rates. Viscosity values at infinite shear rate can be accepted as plastic viscosity

$$
\eta_{\text {inf }}=\mu=\lim _{\dot{\gamma} \rightarrow \infty} \frac{\partial \tau}{\partial \dot{\gamma}} .
$$

In this study, it has been shown that viscosity values are shear dependent for most of the microconcrete mixtures. In order to make comparisons between different samples, a single viscosity value should be defined. For this purpose, viscosity values at maximum allowable shear rate (35 $\mathrm{s}^{-1}$ ) have been accepted as 'plastic viscosity $\left(\mu^{*}\right)$ ' values. Note that, viscosity values became constant for most of the cement paste mixtures at $35 \mathrm{~s}^{-1}$ shear rate (figures 7-9). However, viscosity values were still changing for some other mixtures. For this reason, plastic viscosity notation defined in this study $\left(\mu^{*}\right)$ can be accepted as apparent viscosity values at $35 \mathrm{~s}^{1}$ :

$$
\mu^{*}=\eta_{\dot{\gamma} \rightarrow 35 s^{-1}}
$$

After this section, $\mu^{*}$ will be called as plastic viscosity. Plastic viscosity values of all mixtures have been presented in table 2. Additionally, viscosity (Pa.s) versus shear rate $\left(\mathrm{s}^{-1}\right)$ graphs have been plotted in order to demonstrate the variation of instant viscosity values by shear rate (Figures 7-9). Note that, in order to differentiate varying mixtures, viscosity axis of these graphs has been plotted in logarithmic scale.

\subsection{Determination of hysteresis areas and thixotropic behaviour}

There are different types of thixotropy definitions in literature (Koehler \& Fowler 2004; Westerholm et al 2007; Hanehara \& Yamada 2008). This study focused on the aspect of using ascending and descending flow curve hysteresis areas for the determination of thixotropy. Hysteresis loops of each ascending and descending flow curves have also been plotted by using Herschel-Bulkley model curves. The differential area between each ascending and descending flow curves have been calculated by using rheometer software's formulae

$$
A_{\text {hyst }}=\left[\sum_{i=1}^{n-1} A_{i, u p}\right]-\left[\sum_{i=1}^{n-1} A_{i, \text { down }}\right] \text {, }
$$



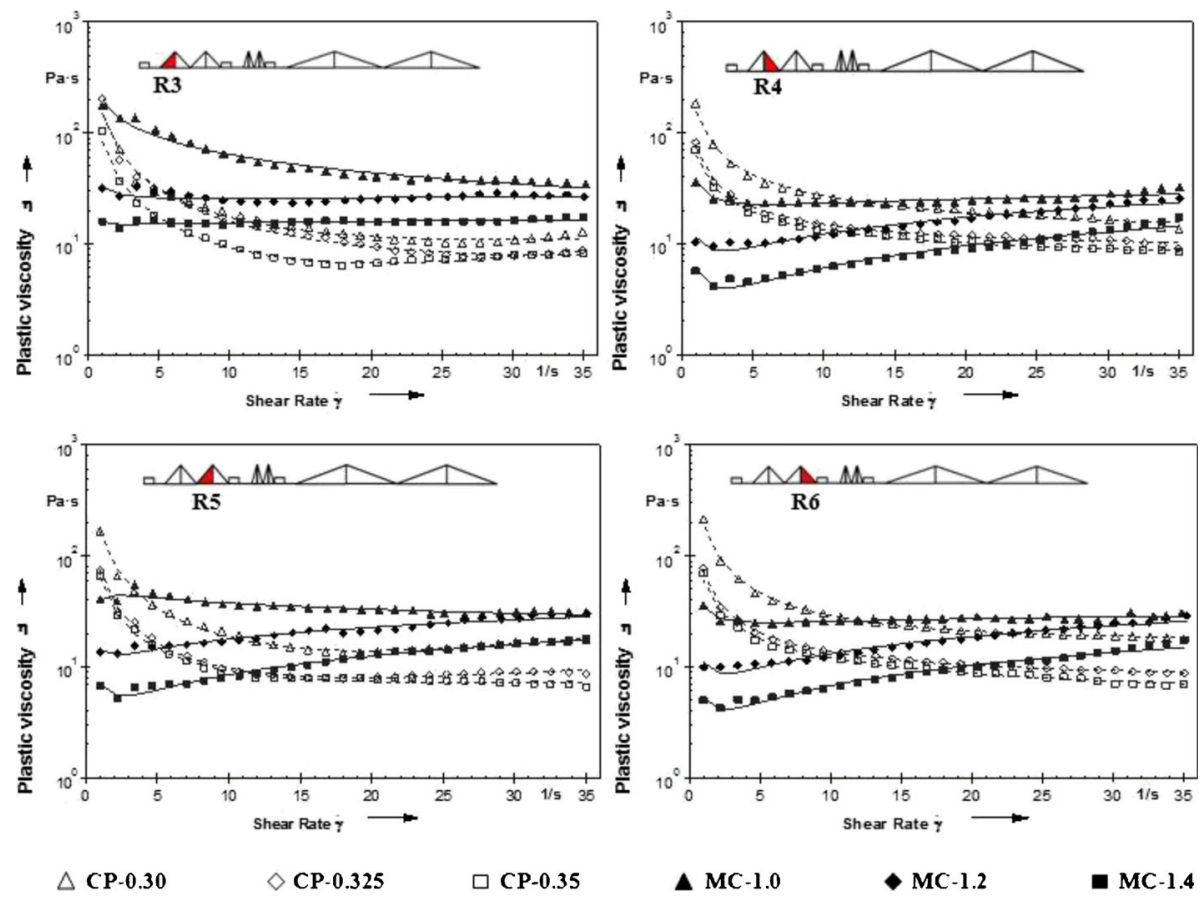

$\Delta$ MC-1.0

$\diamond \mathrm{MC}-1.2$

- MC-1.4

Figure 7. Viscosity curves of each mixture at the normal speed ramps group (R3-R4-R5-R6). (CP: cement paste, 0.30-0.325-0.35 refer to water/cement ratio, MC: micro-concrete, 1.0-1.2-1.4 refer to superplasticizer dosage by weight of cement).

$$
A_{i}=\frac{1}{2}\left[\left(\dot{\gamma}_{i+1}-\dot{\gamma}_{i}\right) \cdot\left(\tau_{i}+\tau_{i+1}\right)\right],
$$

where n: number of data points, $A_{\text {hyst }}$ : Hysteresis area $(\mathrm{Pa} / \mathrm{s}), A_{i, u p}$ : Area under the ascending flow curve $(\mathrm{Pa} / \mathrm{s}), A_{i, \text { down }}$ : Area under the descending flow curve $(\mathrm{Pa} / \mathrm{s}), \tau$ : Yield stress $(\mathrm{Pa})$ and $\dot{\gamma}$ : Shear rate $\left(s^{-1}\right)$.

According to Koehler \& Fowler (2004), hysteresis areas can be used to determine the structural breakdown or structural recovery of materials by shear rate change. Westerholm et al (2007) and Hanehara \& Yamada (2008) postulated that it is possible to investigate the thixotropic behaviour of materials by using their hysteresis areas. However, the shape of hysteresis areas may also change due to fluctuations of flow curves by shear rate as reported in Banfill's (1987) and Baudez's (2006) studies. From this point, Wallevik (2003) reported that thixotropy can be associated with the relative change of a single rheological property as a function of time. Results of experimental studies conducted by Roussel (2005), Ovarlez \& Roussel (2006) and Roussel (2007) showed that hysteresis areas may give a rough idea about the thixotropic behaviour; however, quantitative determination of thixotropy rate is not always certain due to variations of hysteresis loops.

There are three different possibilities for the shape of a hysteresis area (figure 10). This kind of grouping was also mentioned by Banfill \& Saunders (1981). If the ascending curve is under the descending curve, the differential hysteresis area will be negative. The mixtures that exhibits negative hysteresis areas indicate a 'structural recovery' behaviour (figure 10a). According to Koehler \& Fowler (2004), this behaviour may also be called as 'anti-thixotropy'. The second 

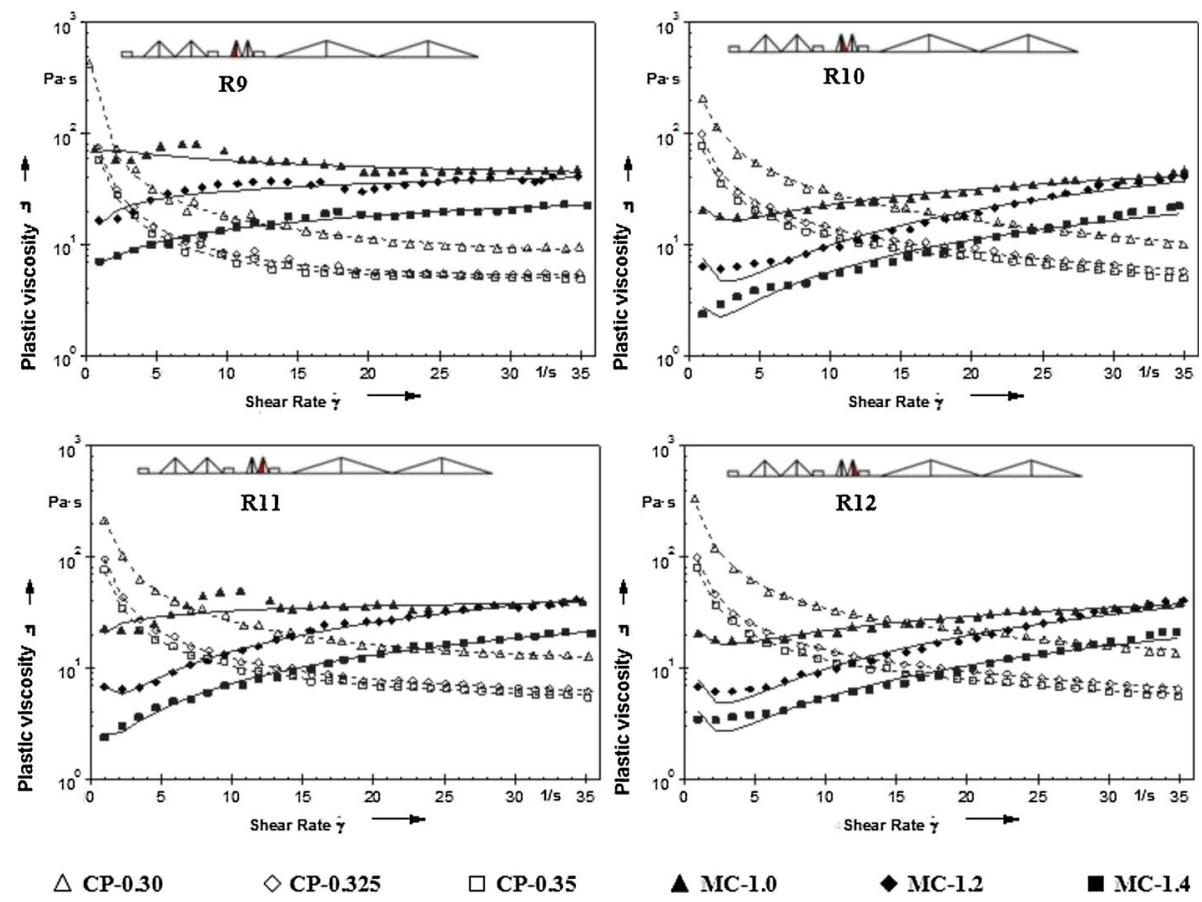

Figure 8. Viscosity curves of each mixture at the high speed ramps group (R9-R10-R11-R12). (CP: cement paste, 0.30-0.325-0.35 refer to water/cement ratio, MC: micro-concrete, 1.0-1.2-1.4 refer to superplasticizer dosage by weight of cement).

possibility is the formation of a positive area where ascending curve is over the descending one. Thixotropic mixtures usually present positive hysteresis areas which indicate a 'structural breakdown' behaviour (figure 10b). In the case of a mixed behaviour, the ascending and descending parts of flow curves at least intersect at a point. For this reason, both positive and negative areas will be formed at the same time. The sign of cumulative areas may be negative or positive (figure 10c). Furthermore, starting and final point of hysteresis loop may not arise from the same origin. The hysteresis loop deviations had been investigated by Wallevik (2005). According to Wallevik (2005), non-recoverable microstructural changes may cause local segregation. Re-building of microstructure and clustering of particles due to surface electrostatic charges at low shear rates may be the main causes of hysteresis loop point deviation. The hysteresis loop areas calculated according to the above mentioned procedure have been presented in table 3 .

\section{Test results and discussion}

\subsection{Flow curve results and comparison of yield values}

Flow curves of cement pastes (hollow boxes) and micro-concrete (solid boxes) mixtures have been presented in figures 4-6. Each figure represents two repetitive sets of ascending and descending shear rate protocols (ramps). The speed of shear rate changes has been notated as normal, high and low for figures 4, 5 and 6, respectively. 

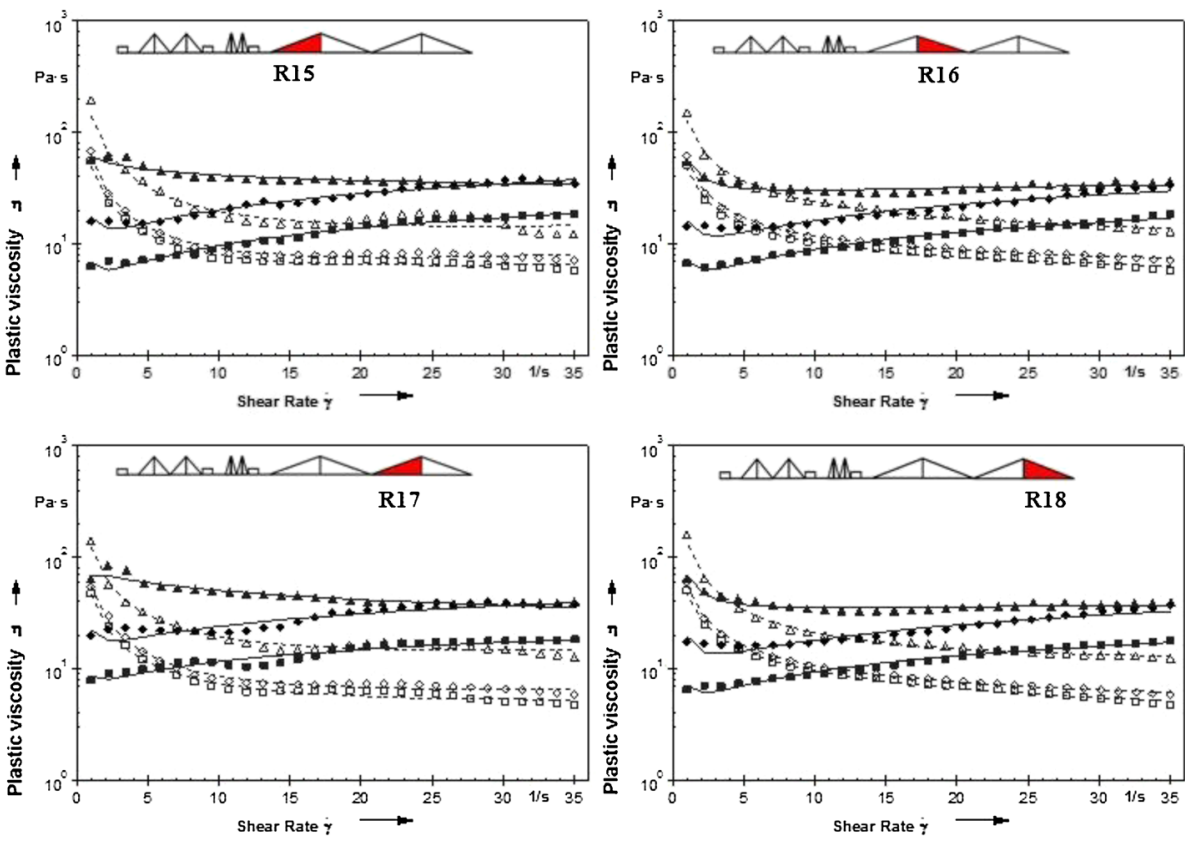

$\triangle \mathrm{CP}-0.30 \quad \diamond \mathrm{CP}-0.325 \quad \square \mathrm{CP}-0.35$

$\triangle \mathrm{MC}-\mathbf{1 . 0}$

$\leftrightarrow \mathrm{MC}-1.2$

MC-1.4

Figure 9. Viscosity curves of each mixture at the low speed ramps group (R15-R16-R17-R18). (CP: cement paste, $0.30-0.325-0.35$ refer to water/cement ratio, MC: micro-concrete, 1.0-1.2-1.4 refer to superplasticizer dosage by weight of cement).

The flow curves of ramps at normal speed shear rate changes (R3, R4, R5 and R6 in figure 3) have been presented in figure 4. In general, a reduction on the slope of flow curves of microconcretes has been observed with the increasing HRWRA dosage. The shapes of ascending flow curves (R3 and R5) of micro-concretes with low HRWRA contents were concave. On the contrary, micro-concretes incorporating high amounts of HRWRAs yielded convex flow curves. Independent from the HRWRA dosage, convex shaped descending flow curves were obtained

Table 2. Plastic viscosity $\left(\mu^{*}\right)$ of mixtures determined by Herschel-Bulkley analyses (Pa.s).

\begin{tabular}{|c|c|c|c|c|c|c|c|c|c|c|c|c|}
\hline \multirow{3}{*}{$\begin{array}{l}\text { Section no } \\
\text { Mixture code }\end{array}$} & 3 & 4 & 5 & 6 & 9 & 10 & 11 & 12 & 15 & 16 & 17 & 18 \\
\hline & \multicolumn{4}{|c|}{$\begin{array}{c}\text { Shear rate acceleration: } \\
\text { normal }\end{array}$} & \multicolumn{4}{|c|}{$\begin{array}{c}\text { Shear rate acceleration: } \\
\text { high }\end{array}$} & \multicolumn{4}{|c|}{$\begin{array}{c}\text { Shear rate acceleration: } \\
\text { low }\end{array}$} \\
\hline & & & a.s) & & & & Pa.s) & & & & $\overline{\text { Pa.s) }}$ & \\
\hline CP- 0.30 & 11 & 16 & 18 & 19 & 9 & 11 & 13 & 15 & 15 & 14 & 15 & 13 \\
\hline CP-0.325 & 8 & 10 & 9 & 9 & 5 & 6 & 6 & 7 & 8 & 7 & 7 & 6 \\
\hline CP-0.35 & 9 & 9 & 7 & 7 & 5 & 5 & 6 & 6 & 7 & 6 & 5 & 5 \\
\hline MC-1.0 & 32 & 28 & 29 & 29 & 45 & 42 & 39 & 38 & 34 & 34 & 36 & 37 \\
\hline MC- 1.2 & 26 & 24 & 28 & 26 & 40 & 37 & 42 & 36 & 38 & 30 & 39 & 33 \\
\hline MC-1.4 & 17 & 14 & 18 & 15 & 23 & 19 & 21 & 18 & 19 & 17 & 18 & 17 \\
\hline
\end{tabular}

*Instantaneous viscosity values at maximum allowable shear rate $\left(35 \mathrm{~s}^{-1}\right)$ are accepted as plastic viscosity values. 


\section{Structural Recovery}
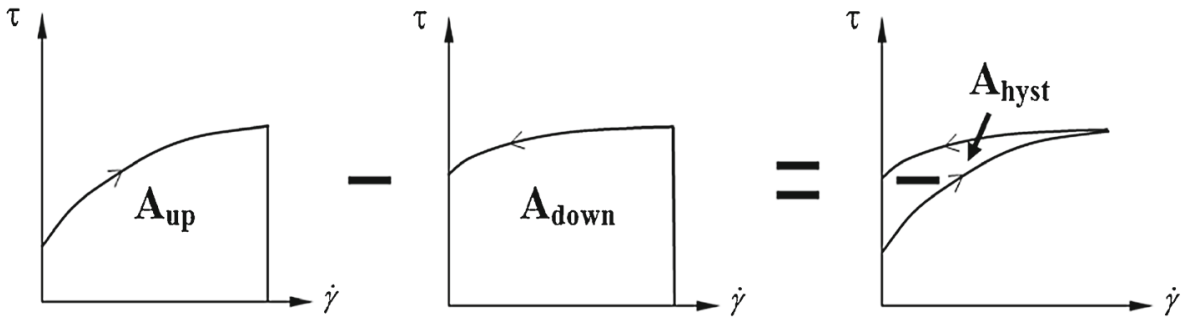

(a)

\section{Structural Breakdown}
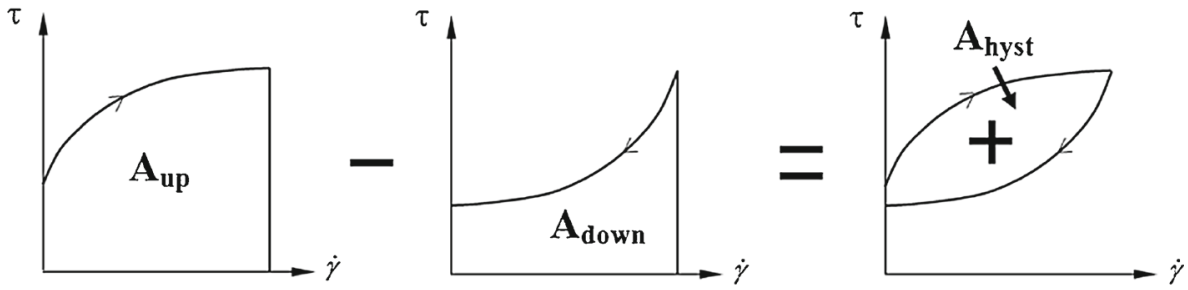

(b)

\section{Mixed behavior}
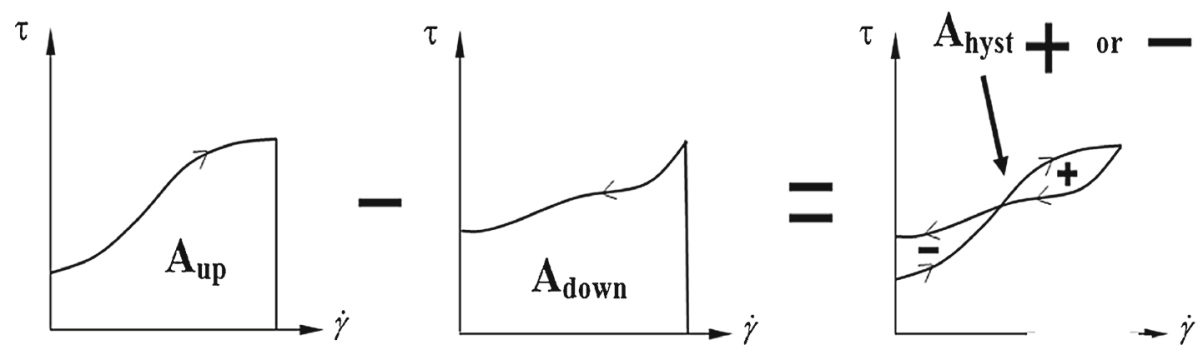

(c)

Figure 10. Hysteresis area types; (a) Structural recovery (anti-thixotropy), (b) Structural breakdown (thixotropy); (c) mixed behaviour.

from all micro-concrete mixtures (R4 and R6). From these results, it is clear that there is a significant flow curve shape difference between the ascending and descending parts of flow curves of micro-concrete in the presence of low amounts of HRWRA. Additionally, the flow curve data stabilized and became more coherent at the descending ramps of shear rate pattern. The yield values of micro-concretes calculated by Herschel-Bulkley analysis were in the order of 0-25 $\mathrm{Pa}$. Note that, due to the shape differences of flow curves at ascending and descending shear rate ramps, the yield values of the same mixture calculated by HB analysis may vary. For example, 
Table 3. The hysteresis loop areas of mixtures $(\mathrm{Pa} / \mathrm{s})$.

\begin{tabular}{|c|c|c|c|c|c|c|c|}
\hline \multirow[b]{3}{*}{ Mixture code } & \multicolumn{6}{|c|}{ Hysteresis loop areas $(\mathrm{Pa} / \mathrm{s})$} & \multirow[b]{3}{*}{ Behaviour* } \\
\hline & $3-4$ & $5-6$ & $9-10$ & $11-12$ & $15-16$ & $17-18$ & \\
\hline & \multicolumn{2}{|c|}{$\begin{array}{c}\text { Shear rate acceleration: } \\
\text { normal }\end{array}$} & \multicolumn{2}{|c|}{$\begin{array}{c}\text { Shear rate acceleration: } \\
\text { high }\end{array}$} & \multicolumn{2}{|c|}{$\begin{array}{c}\text { Shear rate acceleration: } \\
\text { low }\end{array}$} & \\
\hline CP-0.30 & -4202 & -3356 & -3135 & -2785 & -393 & 331 & Structural \\
\hline CP-0.325 & -855 & -958 & -1579 & -706 & -487 & -313 & recovery, \\
\hline CP-0.35 & -1408 & -625 & -1141 & -438 & -494 & -457 & anti-thixotropic \\
\hline MC-1.0 & 11037 & 3163 & 10289 & 3877 & 2711 & 2905 & Structural \\
\hline MC-1.2 & 4633 & 2242 & 6884 & 3121 & 3706 & 4081 & breakdown, \\
\hline MC-1.4 & 3207 & 1323 & 3333 & 1307 & 843 & 1102 & thixotropic \\
\hline
\end{tabular}

*According to Koehler \& Fowler (2004)

due to the concave shape of flow curves of MC-1.0 mixture at the R3 and R5 ramps, negative yield values were calculated. Since negative yield values are physically meaningless, they have been assumed as 0 (table 1). If the previous mentioned exception is neglected, it may be generalized that yield values of micro-concretes decreased with the increasing HRWRA dosage. The most stabilized flow curve ramp is the second shear rate descending ramp (R6). The yield values of MB1.0, MB1.2 and MB1.4 mixtures at this ramp were 17, 8 and $4 \mathrm{~Pa}$, respectively.

The flow curves of cement paste mixtures have been presented at the same figure by hollow boxes (figure 4). Both ascending and descending curves were usually convex or linear. Linear curves were expectable since cement pastes have usually been characterized by Bingham model. The path differences between the ascending and descending curves were also lower compared to micro-concrete mixtures. The yield values of cement paste mixtures vary between 49 and 190 $\mathrm{Pa}$. There is a strong inverse relationship between the $\mathrm{W} / \mathrm{C}$ ratio and yield values (table 1 ). Yield values of cement pastes of CP-0.30, CP-0.325 and CP-0.35 derived from the flow curve at R6 ramp were 190, 64 and $49 \mathrm{~Pa}$, respectively. Note that, the yield values of cement paste mixtures were ten-fold higher than that of micro-concretes. Since low yield values are preferred for selfflowability, experimental findings confirmed the suitability of micro-concrete mixtures for the easily flowable matrix applications.

Flow curves presented in figure 5 were obtained with higher speed of shear rate changes (R9, $\mathrm{R} 10, \mathrm{R} 11$ and R12 in figure 3). In general, the fluctuation of data became more pronounced at high speed shear rate changes. This is possibly due to the effect of acceleration of ball spindle while dragging in the mixture. Ball spindle slightly swings in the mixture during rotation. Except few data fluctuation, similar curve behaviour has been observed for micro-concrete and cement paste mixtures with a lower accuracy (R9-R12). Flow curves of most of the mixtures were stabilized again at the second descending part of this shear rate period (R12). The yield values derived from the flow curves at this ramp were 15, 7 and $4 \mathrm{~Pa}$ for MC-1.0, MC-1.2 and MC-1.4, respectively. On the other hand, yield values of cement pastes of CP-0.30, CP-0.325 and CP0.35 were 215, 72 and $65 \mathrm{~Pa}$, respectively. These results were usually similar or slightly higher compared to the yield values obtained from normal shear rate speed. Slightly higher yield values were more pronounced for cement paste mixtures.

The last group of flow curves derived from the low speed of shear rate change (R15, R16, R17 and R18 in figure 3) has been presented in figure 6. The fluctuation of flow curve data at the initial ramp (R15) was significant for low W/C ratio cement paste and micro-concrete mixtures incorporating the lowest HRWRA dosage. The most stable flow curves have been obtained from 
the last ramp (R18). The yield values of MC-1.0, MC-1.2 and MC-1.4 mixtures calculated by using the flow curves at this ramp were 47, 15 and $4 \mathrm{~Pa}$, respectively. Note that, while the yield values of MC-1.2 and MC-1.4 mixtures were nearly similar with the previous results, the yield value of MC-1.0 mixture is at least three times higher. It seems that decrease in the acceleration of shear rate improved the structure rebuilding rate of micro-concrete with low HRWRA content. On the other hand, yield values of cement pastes of CP0.30, CP-0.325 and CP-0.35 were 131, 47 and $34 \mathrm{~Pa}$, respectively. Decrease in shear rate acceleration also decreased the yield values obtained from cement pastes independent from W/C ratio when compared with tests performed at normal speed shear rates.

The flow curve results presented in the previous paragraph showed that yield values of microconcretes determined by using Herschel-Bulkley analysis were generally lower than that of cement pastes at different W/C ratios. The possible causes of low yield values of micro-concrete mixtures can be attributed to the addition of HRWRA. Previous studies conducted by Flatt (2004a) and Flatt \& Houst (2001) showed that HRWRAs reduce the yield value by supplying both electrostatic and physical interactions on cement particles. Test results also indicated that yield values were dependent on the speed of shear rate application (acceleration). Higher speeds may increase and low speeds may decrease the yield values of micro-concretes and cement pastes. However, the magnitude of change was strongly related to the mixture proportions (W/C ratio in case of cement pastes and HRWRA dosage in case of micro-concretes).

\subsection{Viscosity curve results and comparison of plastic viscosities}

Viscosity curves of cement pastes (hollow boxes) and micro-concrete (solid boxes) mixtures have been presented in figures 7-9. Similar to flow curves, each figure has been representing two repetitive sets of ascending and descending shear rate protocols (ramps). Note that viscosity axis was in logarithmic scale due to the wide range of viscosity values of different mixtures. The speeds of shear rate changes were notated as normal, high and low for figures 7,8 and 9, respectively.

In general, viscosity curves indicated the shear thinning or shear thickening behaviour of mixtures with increasing shear rate. Instant viscosity values of cement pastes at low shear rates were usually higher (figure 7-9). With the increasing shear rates, viscosity values have been decreased to a limit value. Higher shear rates above this limit, may only cause negligible reduction in viscosity values for most of the cement paste mixtures. Cement paste mixtures representing this behaviour may be classified as shear thinning. The plastic viscosity values (instant viscosities at $35 \mathrm{~s}^{-1}$ ) of cement paste mixtures have been varied between 5 and 19 Pa.s which were inversely proportional to the $\mathrm{W} / \mathrm{C}$ ratios at all flow curve analyses (table 2). For example, the plastic viscosity values of CP-0.30, CP-0.325 and CP-0.35 were 13, 6 and 5 Pa.s, respectively. As a general consideration, decrease in plastic viscosity values may cause stability problems on cement pastes with high W/C ratios. On the other hand, micro-concrete mixtures exhibited both shear thinning and thickening behaviour depending on the HRWRA dosage and the speed of shear rate change (figure 7-9). Additionally, the same micro-concrete mixture may exhibit both shear thinning and thickening behaviour at different shear rate intervals. At the initial stages of analysis (ascending ramp R3), MC-1.0 mixture exhibited a shear thinning viscosity curve (figure 7). Micro-concretes behaviour with higher HRWRA dosages (MC-1.2 and MC1.4) can be classified nearly Newtonian at this ramp (R3). In other words, their viscosity values were approximately similar at increasing shear rates. Descending ramps of the viscosity curves (R4) showed that, viscosity values at low shear rates slightly decreased and then tend to be increased with the increasing 
shear rates (figure 7). The latter behaviour may be classified as 'shear thickening'. Similar shear thickening behaviour of micro-concrete mixtures became more significant at the other ramps of this set (R5, R6). The plastic viscosity values of micro-concretes at normal speed of shear rate changes were in the order of 14-32 Pa.s (table 2).

The viscosity curves of mixture exposed to high speed shear rate change have been presented in figure 8 . The shear thickening behaviour of micro-concretes incorporating high amounts of HRWRA (\%1.2-1.4) became more significant in this case. The resistance of micro-concrete mixture against shear increased at higher shear rates. Similar promoting effect of HRWRA dosage on shear thickening behaviour of cement pastes have been previously reported by Cyr et al (2000), Mouret \& Cyr (2003); Artelt \& Garcia (2008). Plastic viscosity values measured from the viscosity curve of the last ramp of this set (R12) were 38, 36 and 18 Pa.s for MC-1.0, MC-1.2 and MC-1.4 mixture respectively.

Viscosity curves presented in figure 9 have been plotted with the data of slowly accelerated shear rate. Shear thickening behaviour was still significant for this case. However, the slopes of viscosity curves were slightly lower than that of shear rate change at high speed. As a result of this lower slope angle, the plastic viscosity values were slightly lower (table 2). However, they were still at least two times higher than that of plain cement paste mixtures with $\mathrm{W} / \mathrm{C}=$ $0.30-0.35$.

The possible causes of observation of shear thinning and thickening behaviour in selfcompacting materials have been comprehensively studied by Feys et al (2008). There are two main theories considered to be applicable to SCMCs. One is based on the formation of clusters and the other is based on grain inertia. After detailed experimental investigations, Feys et al (2008) concluded that theory of cluster formation is more applicable. According to this theory, shear thickening is a result of the formation of clusters. Shear thickening starts at a certain critical shear stress at which the hydrodynamic forces between the particles dominate the repulsive forces, causing particles to stick together temporarily. This shear thickening effect is a fully reversible, purely hydrodynamic phenomenon. Increasing HRWRA dosages causes an increase in the intensity of shear thickening and a decrease in critical shear stress has been observed for the concretes tested. This is most probably caused by the higher dispersion caused by the HRWRA, resulting in a larger amount of particles available for shear thickening as previously reported in Feys et al (2009). On the other hand, Lombois-Burger et al (2008) showed that depending on the shear rate, hydrodynamic lubrication may or may not avoid direct contact between solid particles of the matrix. This dependency significantly affected by the adsorbed and non-adsorbed parts of the polymer. If there is available polymer in the interstitial solution (enough dosage) shear thickening behaviour may be observed.

As a general statement, higher plastic viscosity values and typical shear thickening behaviour of SCMCs compared to shear thinning behaviour of cement pastes are the distinguishing properties of these materials. From the view point of stability and solid holding capacity (in case of any fibre incorporation) micro-concretes with higher viscosities at high shear rates seem more suitable with the advantages of above mentioned rheological properties. Another advantage of SCMCs that is observed at the stage of rheological testing was their pore filling ability against cement paste mixtures as comparatively visualized in figure 11. The rotating ball shoulder's dragging marks (shown with arrows in figure 11a) closed after a while in the case of SCMC series which indicate this material's filling ability. However, dragging marks were always visible in the case of cement paste series which indicate that neat cement pastes were unable to fill the pores after disturbing by the rotating ball's shoulder. One can consider that up to a certain extent, addition of both water and HRWRA may cause enhanced mouldability for the mix. This is due 


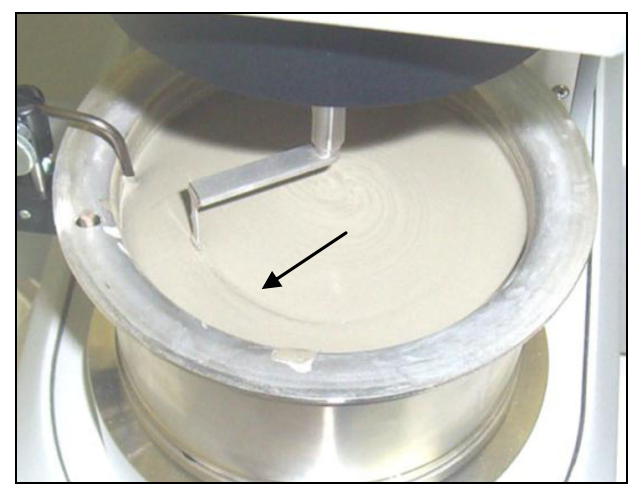

(a)

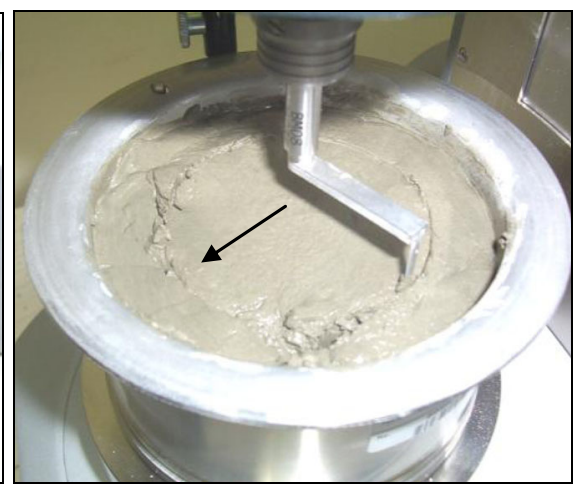

(b)

Figure 11. SCMC with disappearing dragging marks while the ball shoulder rotates (a) and cement paste mixture with permanently visible dragging marks, (b) at the stage of rheological testing with BMS.

to the viscosity reducing effect of these two materials, but as can be seen from figure 11, actual behaviour of the fresh mix could be different from the view point of efficient filling ability.

\subsection{Hysteresis areas and thixotropic behaviour}

Hysteresis areas may give a rough idea about the thixotropy. However, quantitative determination of its rate may not always reflect the thixotropic behaviour due to the shape variations of hysteresis loops. The hysteresis areas of cement paste mixture calculated by using formulae (4) have been listed in table 3. Additionally, the direction of formation of each hysteresis loop areas (by arrows) and resultant hysteresis area have been presented in figures 12-14. Figures have been grouped according to their shear rate application speeds.

In general, the ascending curves of cement paste mixture were under the descending curves. For this reason, negative hysteresis areas have been obtained from most of the cement paste mixtures (except CP-0.30 at the last hysteresis loop). However, some mixed type hysteresis loops were also observed. There were one or more intersections of curves and the resultant loops were in a ribbon-like structure. According to Koehler \& Fowler (2004), negative hysteresis areas reflect an anti-thixotropic behaviour. Structural recovery was more significant for negative hysteresis areas (Figure 10a). As a general tendency, absolute values of hysteresis areas decrease at each repetition of flow curve ramps. Note that this decrease was independent from the speed of shear rate application in case of cement pastes.

In case of micro-concrete mixtures, positive hysteresis areas were observed (figures 12-14). The ascending curves of micro-concrete mixture were usually above the descending curves with some exceptions at low shear rates. A larger positive hysteresis area indicates a slower recovery after shear, which reflects a high thixotropic behaviour. Structural breakdown was more significant for positive hysteresis areas (figure 10b). Additionally, the absolute values of hysteresis areas were comparatively higher than that of cement paste mixtures at the same ramps. However, as a consequence of continuous mixing, the magnitudes of hysteresis areas decreased at the second repetition of normal and high speed of shear rate applications (figures 12-14). For example, the initial hysteresis loop areas of MC-1.0, MC-1.2 and MC-1.4 mixture at normal speed of shear rate application were $+11037,+4633$ and $+3207 \mathrm{~Pa} / \mathrm{s}$, respectively (initial R3-R4 ramp measurement). However, these areas decreased to $+3163,+2242,+1323 \mathrm{~Pa} / \mathrm{s}$, respectively (at 

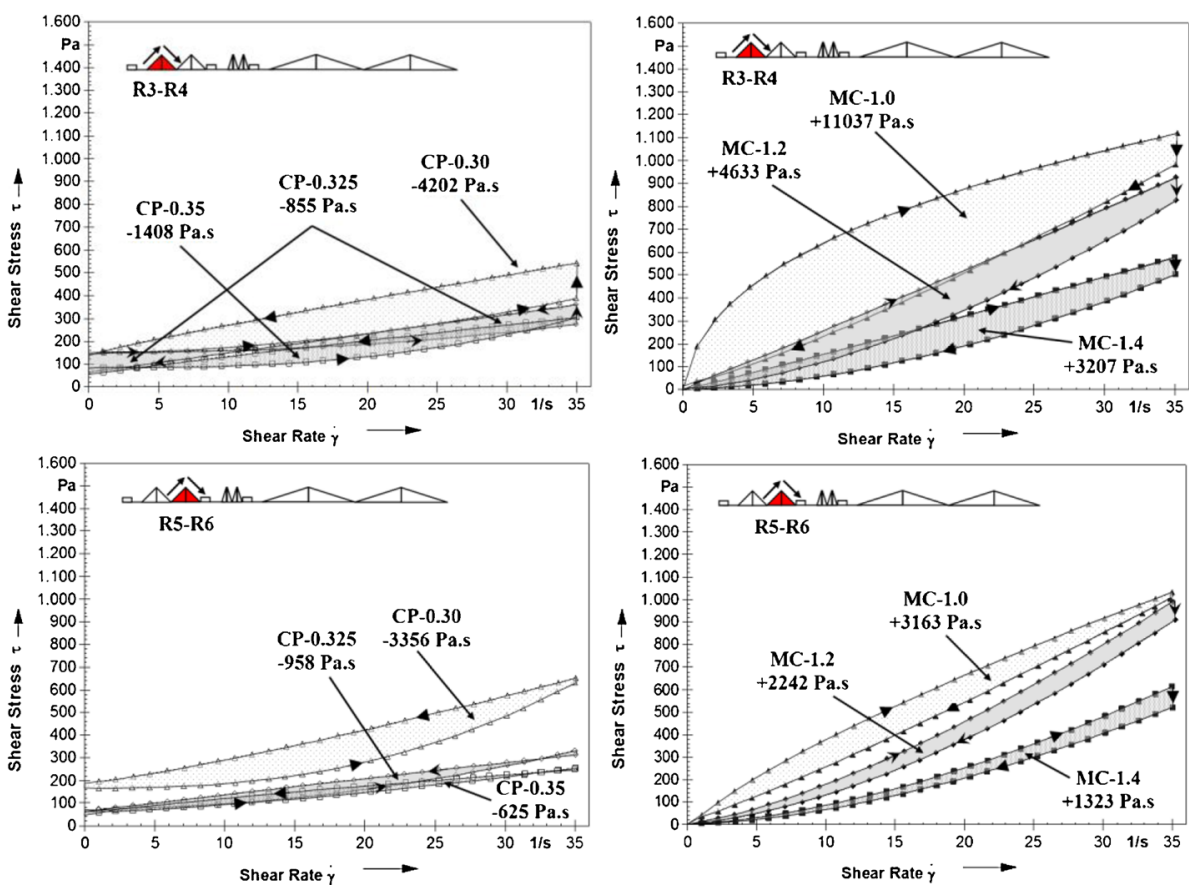

Figure 12. Hysteresis loop formation and hysteresis area differences between cement paste (left) and micro-concretes (right) at normal speed shear rate change (R3-R4 and R5-R6). (CP: cement paste, 0.30$0.325-0.35$ refer to water/cement ratio, MC: micro-concrete, 1.0-1.2-1.4 refer to superplasticizer dosage by weight of cement).

the second repetition of R3-R4 ramp) (figure 12). These results indicated that high initial thixotopic behaviour of micro-concretes decreased at normal and high speed of shear rate application. On the other hand, there was no significant change (even slight increases can be reported) in hysteresis areas at the second repetition in the case of slow speed shear rate application (Figure 13). In addition to shear rate values, the application speed of shear rate significantly affected the thixotropic behaviour of micro-concretes. While slow speeds of application promoted the thixotropic behaviour, higher speeds reduced the thixotropic behaviour.

A generalization from the figures $12-14$ can be concluded as; increasing W/C ratio in case of cement pastes or increasing HRWRA dosage in case of micro-concretes decreased the absolute values of hysteresis areas. Additional water in case of cement pastes or additional HRWRA in case of micro-concrete served as lubricating agents between solid particles of matrices. As a result, the ascending-descending curves of each loop became closer. However, the role of HRWRAs was significantly different from water on the formation of flow curves. While, HRWRs reduced the area of hysteresis loops without causing viscosity loss, addition of water significantly decreased the plastic viscosity values and hysteresis areas. It should be noted that, there were some exceptions where mixed type (ribbon-like) hysteresis areas (figure 12: R3-R4 loop of cement pastes). In these cases, thixotropy increments due to low speed of shear rate increase (figure 14: R15-16 and R17-18 loops of micro-concretes) were also detected. 

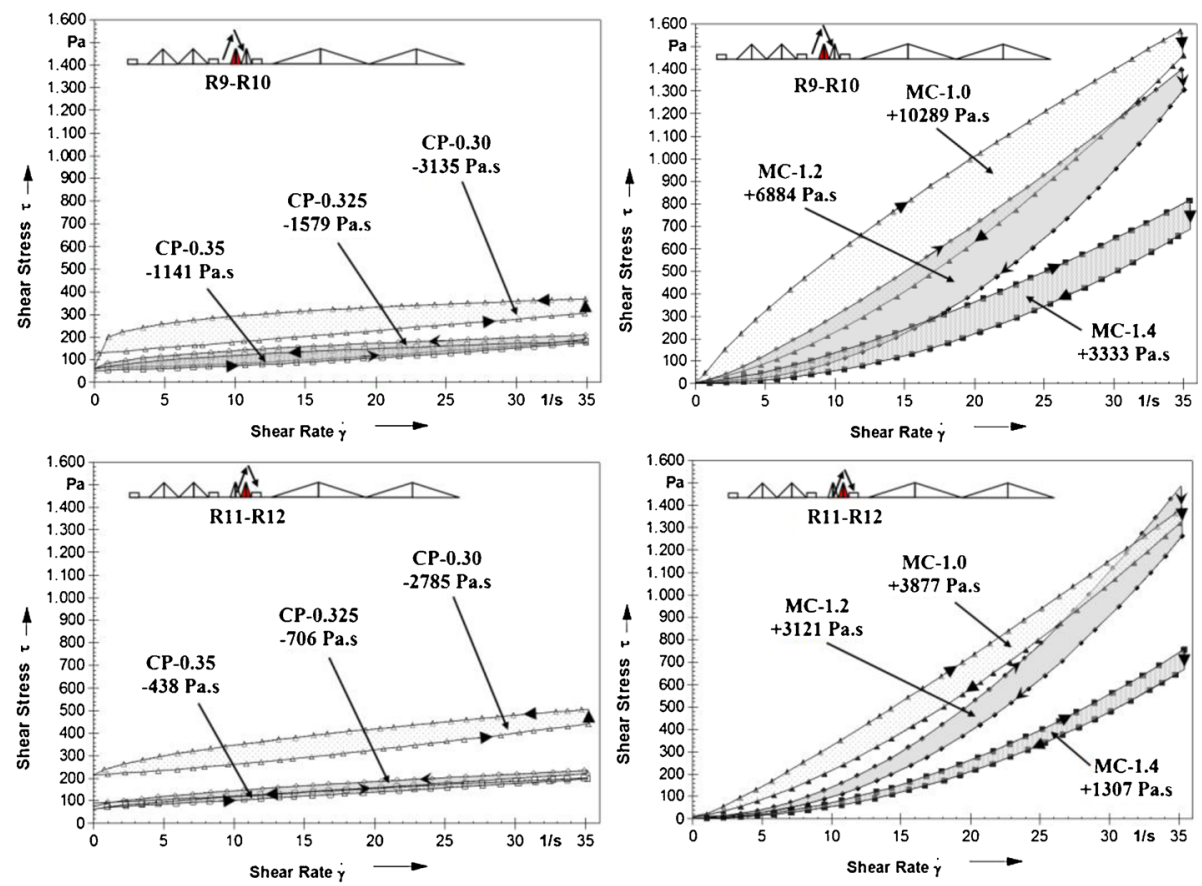

Figure 13. Hysteresis loop formation and hysteresis area differences between cement paste (left) and micro-concretes (right) at high speed shear rate change (R9-R10 and R11-R12). (CP: cement paste, 0.300.325-0.35 refer to water/cement ratio, MC: micro-concrete, 1.0-1.2-1.4 refer to superplasticizer dosage by weight of cement).

\subsection{The role of polycarboxylate based HRWRAs on flow behaviour of cement pastes and micro-concrete mixtures}

Before discussing the role of polycarboxylate based HRWRAs on SCMC rheology, the flow behaviour of cement pastes will be summarized in this section. Cement pastes may be characterized by their relatively high yield values, low plastic viscosities and low thixotropy (negative hysteresis areas) properties. The magnitude of these high yield values and low plastic viscosities are dependent on their $\mathrm{W} / \mathrm{C}$ ratio and speed of shear rate application. The possible causes of this characteristic flow behaviour of cement paste will be discussed by a simple graphical representation in figure 15. As reported by Flatt (2004b), Cement pastes at initial periods of mixing is usually modelled as a mixture of positively and negatively charged solid particles in a colloidal liquid. Due to the high values of $\mathrm{pH}$ and $\mathrm{Ca}^{+2}$ concentration of liquid phase at the initial periods of mixing, the cohesive forces can be related with ionic interactions according to Chougnet et al (2008). The difference between surface charges of cement particles is the main reason to their coagulation behaviour at rest (figure 15a). Flatt \& Bowen (2003) reported that coagulation of cement particles due to the electrostatic forces can be accepted as the origin of high yield values. Chougnet et al (2008), linked this behaviour to the gelation of the suspension due to aggregation of particles. If cement paste mixture is subjected to low shear rates, these electrostatic forces act as barriers against flow direction (figure 15b). After a critical shear rate these barriers breakdown and flow can be observed through shear direction. Loss of electrostatic coagulation related temporary bonding between particles can be accepted as the origin of low viscosity 

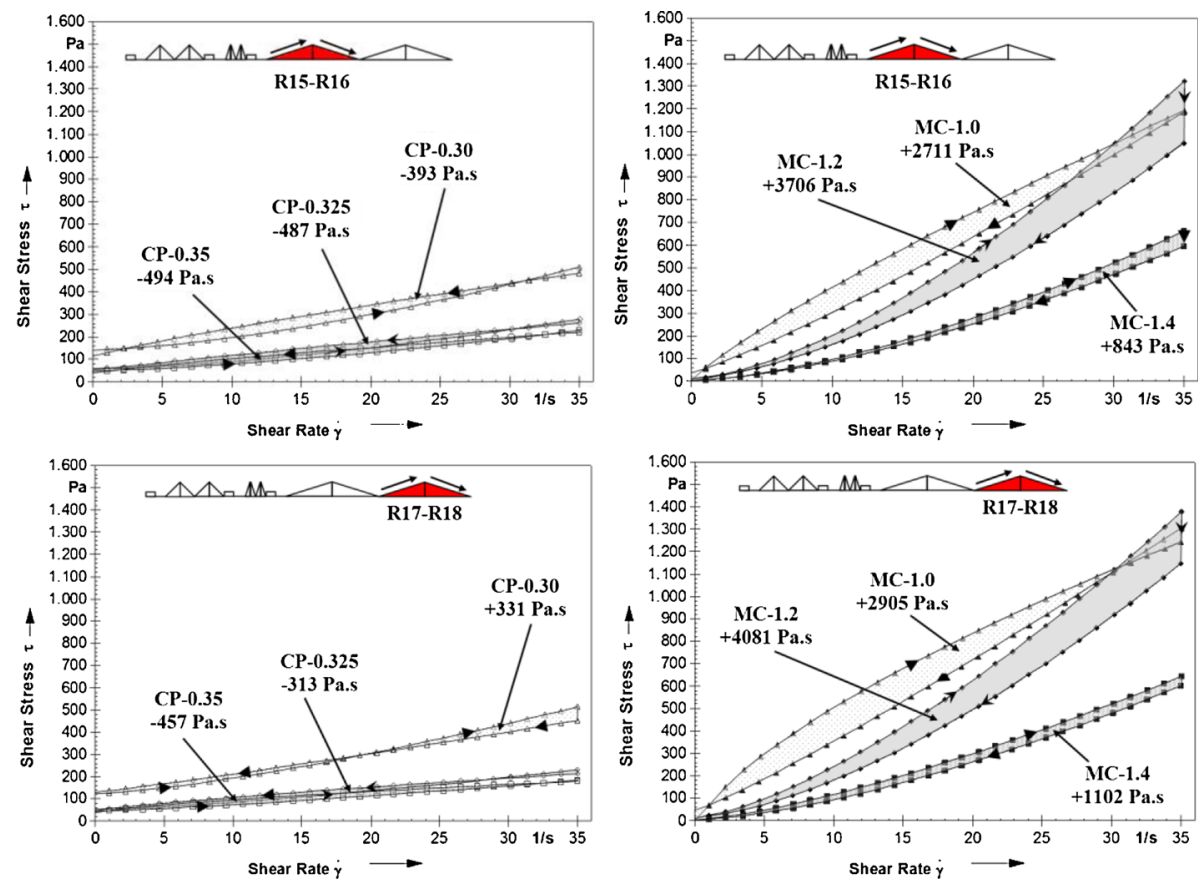

Figure 14. Hysteresis loop formation and hysteresis area differences between cement paste (left) and micro-concretes (right) at low speed shear rate change (R15-R16 and R17-R18). (CP: cement paste, 0.30$0.325-0.35$ refer to water/cement ratio, MC: micro-concrete, 1.0-1.2-1.4 refer to superplasticizer dosage by weight of cement).

(figure 15c). Increasing shear rate enhances the dispersion of particles and progressive crumbling occurs under shear. If the shear rate decreases again, re-coagulation of cement particles can be observed. On the other hand, hysteresis areas of cement pastes can be related with the instant dispersed/coagulated particle ratio of mixtures at definite shear rates. If the shear rate application protocol causes an increase in the dispersed/coagulated particle ratio, lower hysteresis loops will be obtained. From the view point of W/C ratio, it can be generalized that excess amount of liquid phase leads to the formation of smaller hysteresis areas.

In case of micro-concretes at varying polycarboxylate based HRWRA incorporations, comparatively lower yield values and higher plastic viscosities have been measured. Previous studies conducted by Flatt \& Houst (2001), Li et al (2005) and Zingg et al (2008) confirmed this behaviour. Since the polycarboxylate molecules are composed of extremely long polymer chains, these admixtures improve the dispersing ability of particles with a more dominant physical steric hindrance effect in addition to slight electrostatic repulsion (figure 16a). It is possible to verify the dominating effect of steric hindrance (against electrostatic repulsion) on the dispersion ability of cement particles by measuring their zeta potential in the absence and presence of poycarboxylate based HRWRA. Their effect on zeta potential is usually neglectable (Uchikawa et al 1997; Zingg et al 2009). The high performance dispersing ability of polycarboxylate based HRWRAs arising from steric hindrance effect is responsible for the low yield values of microconcretes at extremely low shear rates (nearly stationary). If shear rate increases, free long polymer chains of polycarboxylate molecules (not adsorbed by cement particles) starts to orientate along the shear path (figure 16b). This is a lubricating effect which improves the flow 


\section{Cement paste at rest}

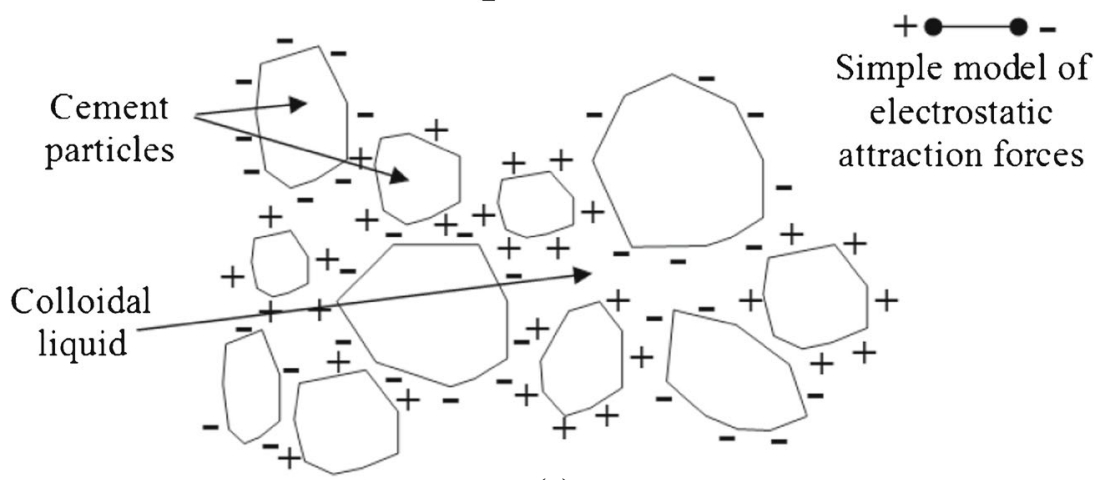

(a)

\section{Cement paste at rest} or under low shear rates

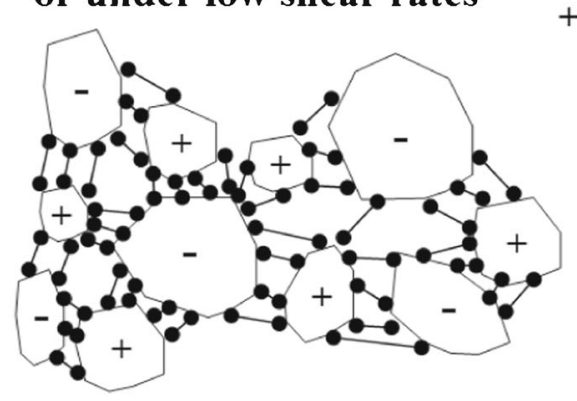

Origin of high yield values
Coagulation of cement particles due to electrostatic forces

(b)

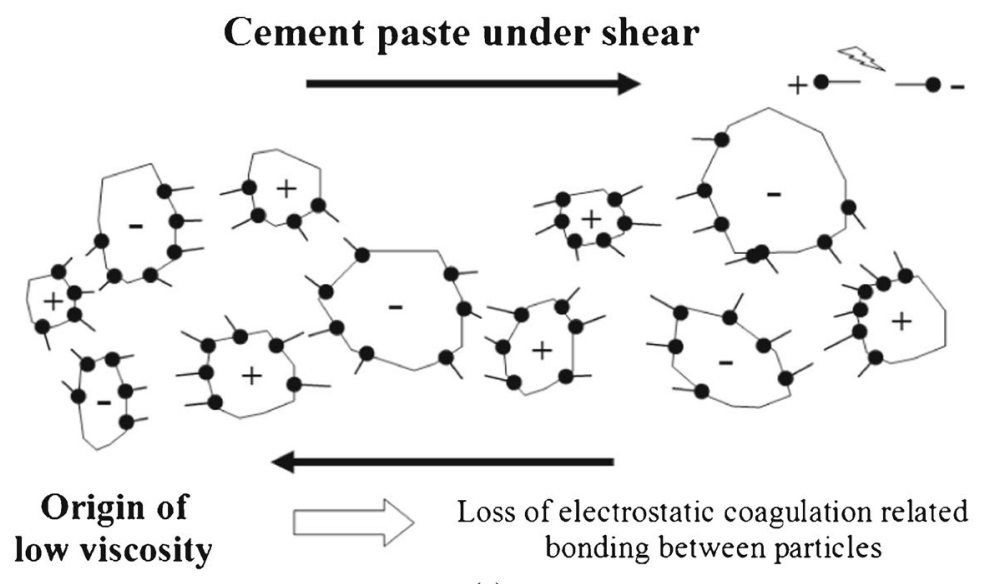

(c)

Figure 15. Rheological behaviour of cement paste mixture at rest (a), under low shear (b) and under high shear rates $(\mathbf{c})$. 
Micro-concrete at rest

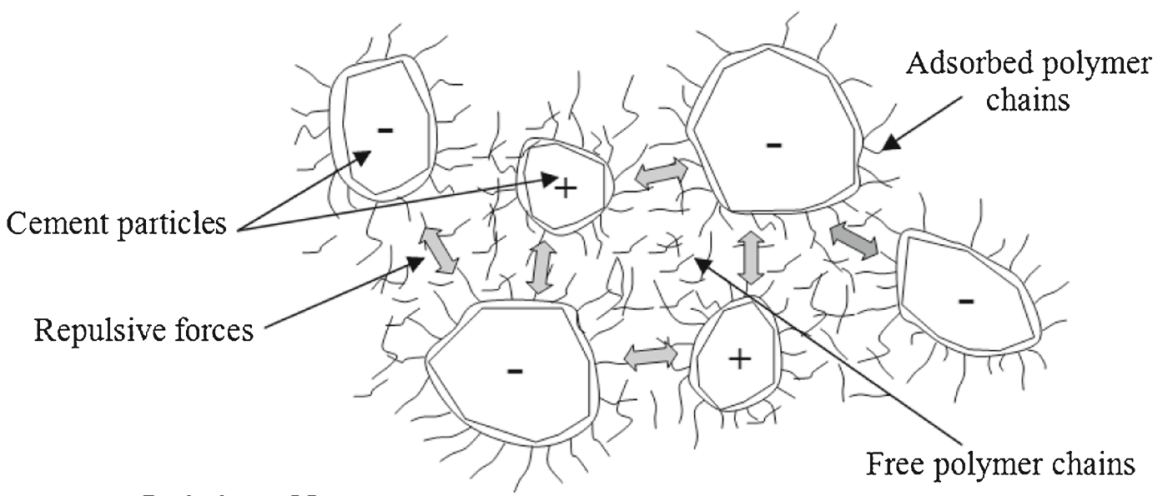

\section{Origin of low yield values}

Steric hindrance effect of polycarboxylate based superplasticizers

(a)

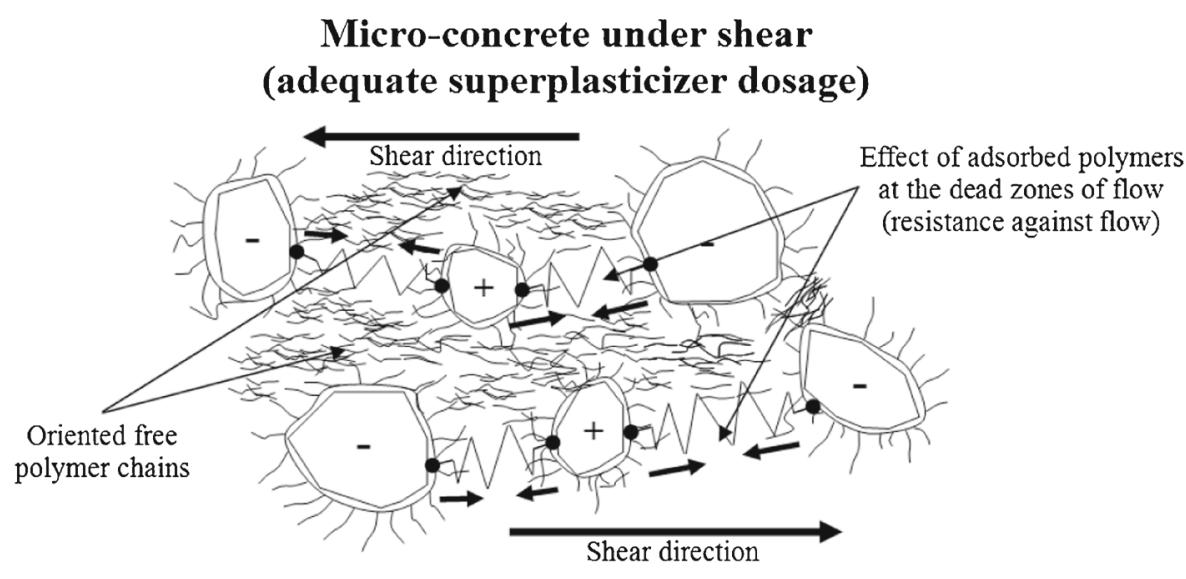

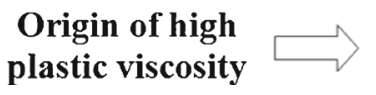

Connection of cement particles due to the clustering of adsorbed polymer chains at the dead zones of flow

(b)

Figure 16. Rheological behaviour of micro-concretes at rest (a) and under shear (b).

behaviour. It should be noted that, the hydrodynamic lubricating role of free polymer molecules in the interstitial fluid phase of cementitious composites was also reported by Lombois-Burger et al (2008). However, the adsorbed polymer chains on the surface of particles attach and obstruct some free polymer chains at dead shear rate zones (perpendicular to flow directions). They can be accepted as weak springs parallel to flow direction (figure 16b). The reverse effect of these local attachments against flow can be accepted as the origin of relatively high plastic viscosity of micro-concretes. This effect also enhances the continuous free flow of micro-concretes (stable 
and collective flow), since no free flowing can be mentioned for cement pastes even at the maximum investigated W/C ratio. Finally, the role of polycarboxylate based HRWRA on hysteresis areas are significantly shear rate speed dependent and difficult to generalize. The orientation of free polymer chains parallel to flow direction at increasing shear rates and results with a positive hysteresis area. Note that the above mentioned cases can be valid in case of adequate polycarboxylate based HRWRA content in the mixture. If these is no free admixture (not adsorbed by cement particles), there will be no free flow. Another factor affecting the efficiency of HRWRAs is the time of addition of admixture into the mixture. Delayed addition usually improves the initial dispersing ability of HRWRAs (Aiad 2003).

Feys et al (2009), explained the shear thickening behaviour of mixtures incorporating HRWRA in a different way. According to them, formation of clusters is the main cause of viscosity increase with the increasing shear rate. Flow is caused by shearing several layers of particles with only interstitial fluid in between the layers. At the onset of shear thickening, the layers start to disappear and shear induced disordering was thought to be the cause of shear thickening. The shear thickening is caused by the high hydrodynamic (lubrication) forces between the particles, overcoming the particle repulsion forces and forming temporary assemblies of particles, named 'hydro-clusters'. In time, particles can join and leave the cluster, causing this phenomenon to be transient.

Rheological measurements performed in this study generally showed that micro-concretes exhibit some potential advantages and disadvantages. While low yield values and moderate viscosities are the main advantages of micro-concretes, shear thickening behaviour may be considered as a potential industrial problem in terms of casting such special mixtures as reported by Toussaint et al (2009). For example, fresh SCMC will be exposed to high shear rates while pumping and if shear thickening effect is dominant energy requirements of placement work will considerably increase in that case. From this point of view, an optimization between HRWRA dosage and water content is necessary to improve the rheological properties of SCMC without causing significant shear thickening effect.

\section{Conclusions}

(i). The rheological behaviour of cement pastes and micro-concretes were significantly different in terms of yield value and plastic viscosity values. While cement pastes investigated in this study possessed high yield values (34-217 Pa) and very low viscosities (5-19 Pa.s), comparatively high viscosities (17-45 Pa.s) and low yield values (0-47 Pa) have been obtained from micro-concretes. The comparatively high viscosity of microconcretes may improve the solid (for example, fibre or coarse aggregate incorporation) holding capacity of this composite at a stable condition without loosing self flowability. On the other hand, comparatively low viscosities of cement pastes may bring out segregation susceptibility under shear.

(ii). The rheological properties of micro-concretes such as yield value, plastic viscosity and hysteresis areas were highly dependent to the speed of shear rate application. Application of accelerated shear rate usually caused flow curve data fluctuations and reduced the accuracy of results. Due to this reason, speed of shear rate application should be limited. The dependency of cement paste on the speed of shear rate application was comparatively much lower. 
(iii). In general, cement paste mixtures investigated in this study showed shear thinning and micro-concretes showed shear thickening characteristics. In case of micro-concretes, effect of shear thickening became more significant with increasing HRWRA dosages.

(iv). The plastic viscosity values of micro-concretes increased with the increasing shear rates. This behaviour may be attributed to the formation of local attachments due to the coagulation of long polymer chains of polycarboxylate based HRWRAs at the dead zones of flow.

(v). Negative hysteresis areas (between -4202 and $-313 \mathrm{~Pa} / \mathrm{s}$ ) have been obtained from cement pastes. Absolute values of hysteresis areas decreased with the increasing W/C ratio. On the other hand, positive hysteresis areas (between +11037 and $843 \mathrm{~Pa} / \mathrm{s}$ ) have been obtained from micro-concretes. The ascending curve was over the descending curve for most of the flow curves which can be attributed to their thixotropic behaviour.

\section{Acknowledgement}

The financial support from the Scientific and Technical Research Council of Turkey (TUBITAK) under grant no. MAG 108M062 is acknowledged.

\section{References}

Aiad I 2003 Influence of time addition of superplasticizers on the rheological properties of fresh cement pastes. Cem. Concr. Res. 33(8): 1229-1234

Artelt C and Garcia E 2008 Impact of superplasticizer concentration and of ultra-fine particles on the rheological behaviour of dense mortar suspensions. Cem. Concr. Res. 38(5): 633-642

Azeredo G, Morel J-C and Lamarque C-H 2008 Applicability of rheometers to characteriz-ing earth mortar behaviour. Part I: Experimental device and validation. Mater. Struct. 41(8): 1465-1472

Banfill P and Frias M 2007 Rheology and conduction calorimetry of cement modified with calcined paper sludge. Cem. Concr. Res. 37(2): 184-190

Banfill P F G 1987 Feasibility study of a coaxial cylinders viscometer for mortar. Cem. Concr. Res. 17(2): 329-339

Banfill P F G and Saunders D C 1981 On the viscometric examination of cement pastes. Cem. Concr. Res. 11(3): 363-370

Barrak M, Mouret M and Bascoul A 2009 Self-compacting concrete paste constituents: Hierarchical classification of their influence on flow properties of the paste. Cem. Concr. Comp. 31(1): 12-21

Baudez J-C 2006 About peak and loop in sludge rheograms. J. Env. Management 78(3): 232-239

Chougnet A, Palermo T, Audibert A and Moan M 2008 Rheological behaviour of cement and silica suspensions: Particle aggregation modelling. Cem. Concr. Res. 38(11): 1297-1301

Cyr M, Legrand C and Mouret M 2000 Study of the shear thickening effect of superplasticizers on the rheological behaviour of cement pastes containing or not mineral additives. Cem. Concr. Res. 30(9): $1477-1483$

Felekoglu B 2007 Utilisation of high volumes of limestone quarry wastes in concrete industry (selfcompacting concrete case). Resources, Conservation and Recycling 51(4): 770-791

Felekoglu B 2009 High performance micro-concrete design, Ph.D. Thesis, Dokuz Eylul University. The Graduate School of Natural and Applied Sciences, 428 p. (in Turkish)

Felekoglu B and Sarıkahya H 2008 Effect of chemical structure of polycarboxylate-based superplasticizers on workability retention of self-compacting concrete. Constr. Build Mat. 22(9): 1972-1980

Ferraris C F 1999 Measurement of the rheological properties of cement paste: A new approach. In: Cabrera, J G and Rivera, R R (Eds.) Symposium on the role of admixtures in high performance concrete, Proceedings of RILEM International, Monterrey, Mexico pp. 333-342 
Feys D, Verhoeven R and Schutter G D 2008 Fresh self compacting concrete: a shear thickening material. Cem. Concr. Res. 38(7): 920-929

Feys D, Verhoeven R and Schutter G D 2009 Why is fresh self-compacting concrete shear thickening? Cem. Concr. Res. 39(6): 510-523

Flatt R J 2004a Towards a prediction of super-plasticized concrete rheology. Mater. Struct. 37(5): 289-300

Flatt R J 2004b Dispersion forces in cement suspensions. Cem. Concr. Res. 34(3): 399-408

Flatt R J and Bowen P 2003 Electrostatic repulsion between particles in cement suspensions: Domain of validity of linearized Poisson-Boltzmann equation for nonideal electrolytes. Cem. Concr. Res. 33(6): 781-791

Flatt R J and Houst Y F 2001 A simplified view on chemical effects perturbing the action of superplasticizers. Cem. Concr. Res. 31(8): 1169-1176

Hanehara S and Yamada K 2008 Rheology and early age properties of cement systems. Cem. Concr. Res. 38(2): 175-195

Kaufmann J P, Winnefeld F, Hesselbarth D and Trindler W 2006 Evaluation of the consistency of fiber reinforced cementitious composites. Mater. Struct. 39(6): 645-654

Koehler E P and Fowler D W 2004 Development of a Portable Rheometer for Fresh Portland Cement Concrete. Aggregates Foundation for Technology, Research and Education (AFTRE) Research Report, No. ICAR-105, August, $306 \mathrm{p}$

Kong H-J, Bike S G and Li V C 2003 Development of a Self-Consolidating Engineered Cementitious Composite Employing Electrosteric Dispersion/Stabilization. Cem. Concr. Comp. 25(3): 301-309

Li C-Z, Feng N-Q, Li Y-D and Chen R-J 2005 Effects of polyethlene oxide chains on the performance of polycarboxylate-type water-reducers. Cem. Concr. Res. 35(5): 867-873

Li V C 2002 American Concrete Institute Special Publication on Concrete: Material Science to Applications SP. Advances in ECC Research 206-23: 373-400

Li V C, Kong H J and Chan Y W 1998 Development of Self-Compacting Engineered Cementitious Composites. In: Proceedings of International Workshop on Self-Compacting Concrete, Kochi, Japan, pp.4659

Li V C, Wu C, Wang S, Ogawa A and Saito T 2002 Interface Tailoring for Strain-Hardening Polyvinyl Alcohol- Engineered Cementitious Composite (PVA-ECC). ACI Materials J. 99(5): 463-472

Lombois-Burger H, Colombet P, Halary J L and Van Damme H 2008 On the frictional contribution to the viscosity of cement and silica pastes in the presence of adsorbing and non adsorbing polymers. Cem. Concr. Res. 38(11): 1306-1314

Mazanec O, Lowke D and Schieß1 P 2010 Mixing of high performance concrete: effect of concrete composition and mixing intensity on mixing time. Mater. Struct. 43(3): 357-365

Mouret M and Cyr M 2003 A discussion of the paper 'The effect of measuring procedure on the apparent rheological properties of self-compacting concrete' by Geiker M R Brandl M, Thrane L N, Bager D H and Wallevik O (eds.) Cem. Concr. Res. 33(11): 1901-03

Naaman A E and Reinhardt H W 2006 Proposed classification of HPFRC composites based on their tensile response. Mater. Struct. 39(5): 547-555

Ovarlez G and Roussel N 2006 A physical model for the prediction of lateral stress exerted by self-compacting concrete on formwork. Mater. Struct. 39(2): 269-79

Papo A and Piani L 2004 Flow Behaviour of Fresh Portland Cement Pastes. Particulate Sci. Technol. 22(2): 201-212

Roussel N 2005 Steady and transient flow behaviour of fresh cement pastes. Cem. Concr. Res. 35(9): 16561664

Roussel N 2006 A thixotropy model for fresh fluid concretes: theory, validation and applications. Cem. Concr. Res. 36(10): 1797-1806

Roussel N 2007 The LCPC BOX: A cheap and simple technique for yield stress measurements of SCC. Mater. Struct. 40(9): 889-896

Sahmaran M, Lachemi M, Hossain K M A, Ranade R and Li V C 2009 Influence of aggregate type and size on ductility and mechanical properties of engineered cementitious composites. ACI Materials J. 106(3): 308-316 
Schatzmann M 2005 Rheometry for large particle fluids and debris flows. PhD Thesis. Eidgenössische Technische Hochschule ETH Zürich, Nr.16093: 192pp

Schatzmann M, Bezzola G R, Fischer P, Windhab E J and Minor H E 2004 The ball measuring system a new rheometric tool to determine the flow curve of large particle material. Swiss Group of Rheology Annual Meeting, Poster Session, Lausanne/Switzerland

Schatzmann M, Fischer P and Bezzola G R 2003 Rheological behaviour of fine and large particle suspensions. ASCE J. Hydraul. Eng. 129(10): 796-803

Toussaint F, Roy C and Jézéquel P-H 2009 Reducing shear thickening of cement-based suspensions. Rheol. Acta. 48(8): 883-895

Uchikawa H, Hanehara S and Sawaki D 1997 The role of steric repulsive force in the dispersion of cement particles in fresh paste prepared with organic admixture. Cem. Concr. Res. 27(1): 37-50

Wallevik J E 2003 Rheology of particle suspensions: Fresh concrete, mortar and cement paste with various types of lignosulfonates. Ph.D. Thesis, Norwegian University of Science and Technology, $397 \mathrm{pp}$

Wallevik J E 2005 Thixotropic investigation on cement paste: Experimental and numerical approach. $J$. Non-Newtonian Fluid Mech. 132(1-3): 86-99

Westerholm M, Lagerblad B and Forssberg E 2007 Rheological properties of micromortars containing fines from manufactured aggregates. Mater. Struct. 40(6): 615-625

Yang E -H, Sahmaran M, Yang Y and Li V C 2009 Rheological Control in Production of Engineered Cementitious Composites. ACI Materials J. 106(4): 357-366

Zingg A, Winnefeld F, Holzer L, Pakusch J, Becker S and Gauckler L 2008 Adsorption of polyelectrolytes and its influence on the rheology, zeta potential, and microstructure of various cement and hydrate phases. J. Colloid and Interface Sci. 323(2): 301-312

Zingg A, Winnefeld F, Holzer L, Pakusch J, Becker S, Figi R and Gauckler L 2009 Interaction of polycarboxylate-based superplasticizers with cements containing different $\mathrm{C}_{3} \mathrm{~A}$ amounts. Cem. Concr. Comp. 31(3): 153-162 